\title{
A STRUCTURED COAGULATION-FRAGMENTATION EQUATION IN THE SPACE OF RADON MEASURES: UNIFYING DISCRETE AND CONTINUOUS MODELS
}

\author{
Azmy S. ACKLeH ${ }^{1, *}$, RAiney Lyons $^{1}$ And Nicolas SaintieR ${ }^{2}$
}

\begin{abstract}
We present a structured coagulation-fragmentation model which describes the population dynamics of oceanic phytoplankton. This model is formulated on the space of Radon measures equipped with the bounded Lipschitz norm and unifies the study of the discrete and continuous coagulationfragmentation models. We prove that the model is well-posed and show it can reduce down to the classic discrete and continuous coagulation-fragmentation models. To understand the interplay between the physical processes of coagulation and fragmentation and the biological processes of growth, reproduction, and death, we establish a regularity result for the solutions and use it to show that stationary solutions are absolutely continuous under some conditions on model parameters. We develop a semi-discrete approximation scheme which conserves mass and prove its convergence to the unique weak solution. We then use the scheme to perform numerical simulations for the model.
\end{abstract}

Mathematics Subject Classification. 35L60, 35Q92, 92D25.

Received January 25, 2021. Accepted September 23, 2021.

\section{INTRODUCTION}

Coagulation-fragmentation equations have been used in many applications in physics, chemistry and biology. In particular, they receive much attention in the study of the population dynamics of phytoplankton $[1,3,7,11$, $15,36,37,53]$, which is a vital member of the oceanic ecosystem. Coagulation-fragmentation equations are useful in this application as phytoplankton populations are often modeled as a collection of particles which are held together via an organic glue. Thus, particles can either stick together to form a cell of larger size (coagulate) or fracture off into cells of smaller size (fragment). Coagulation-fragmentation models are often set with either a continuous size structure $[3,15,37]$ or a discrete size structure $[10,16,43]$.

The first discrete coagulation model in the form of a system of differential equations was introduced by Smoluchowski in his seminal work [55]. In [13], Blatz and Tobolsky added discrete fragmentation terms to the model. For completeness, we provide a discrete coagulation-fragmentation equation which encompasses the

Keywords and phrases. Coagulation-fragmentation equations, structured populations, non-negative Radon measures, BoundedLipschitz norm, semi-discrete schemes, conservation of Mass.

1 Department of Mathematics, University of Louisiana at Lafayette, Lafayette, LA 70504, USA.

2 Departamento de Matemática, Facultad de Ciencias Exactas y Naturales, Universidad de Buenos Aires, (1428) Pabellón I, Ciudad Universitaria, Buenos Aires, Argentina.

*Corresponding author: azmy. ackleh@louisiana.edu 
models presented in $[13,55]$

$$
\left\{\begin{array}{l}
\frac{\mathrm{d}}{\mathrm{d} t} u_{l}(t)=\frac{1}{2} \sum_{i=1}^{l-1} \kappa_{i, l-i} u_{i}(t) u_{l-i}(t)-\sum_{i=1}^{\infty} \kappa_{i, l} u_{i}(t) u_{l}(t)+\sum_{i \geq l} b_{l, i} a_{i} u_{i}(t)-a_{l} u_{l}(t) \\
u_{l}(0)=u_{l, 0} .
\end{array}\right.
$$

Here, $u_{i}(t)$ represents the number of particles of the $i$ th size at time $t, \kappa_{i, j}$ represents the rate at which particles of the $i$ th and $j$ th size coagulate, $a_{i}$ represents the global fragmentation rate of particles of the $i$ th size, and $b_{i, j}$ represents the rate at which particles of the $j$ th size fragment to particles of the $i$ th size. In subsequent works, Müller [49] extend the discrete coagulation terms to a continuous setting in the form of an integro-differential equation. In a similar fashion, Melzak [48] extended the fragmentation terms to a continuous setting. In [3], Ackleh and Fitzpartick introduced the coagulation equations in the context of size-structured population where biological processes including birth, death and growth were modeled, and the fragmentation equation were added to this size-structured model by Ackleh in [1]. These models take the form of a nonlinear nonlocal first-order hyperbolic differential equation with a nonlocal boundary condition. All together, these continuous models can be covered under the following partial differential equation:

$$
\left\{\begin{array}{lr}
\partial_{t} u+\partial_{x}(g(t, u) u)+d(t, u) u=K[u]+F[u], & (t, x) \in(0, T) \times(0, \infty) \\
g(t, u)(0) u(0)=\int_{\mathbb{R}^{+}} \beta(t, u)(y) u(y) \mathrm{d} y, & t \in[0, T] \\
u(0, x)=u_{0}(x), &
\end{array}\right.
$$

where

$$
K(u)(x)=\frac{1}{2} \int_{0}^{x} \kappa(y, x-y) u(x-y) u(y) \mathrm{d} y-u(x) \int_{0}^{\infty} \kappa(y, x) u(y) \mathrm{d} y
$$

and

$$
F(u)(x)=\int_{x}^{\infty} b(y, x) a(y) u(y) \mathrm{d} y-a(x) u(x) .
$$

Analogous to the discrete equation, $u(t, x)$ represents the density of individuals of size $x$ at time $t, \kappa(x, y)$ represents the rate at which particles of size $x$ and $y$ coagulate, $a(x)$ represents the global fragmentation rate of particles of size $x$, and $b(y, x)$ represents the rate at which particles of size $y$ fragment into particles of size $x$. Additionally, the functions $g, d$, and $\beta$ represent the growth, death, and birth functions respectively. Each of these functions is influenced by time and density of the population.

Throughout the literature, there are a variety of assumptions on the coagulation kernel. Common assumptions include: the kernel being bounded by some combination of linear functions [10,33]; some ratio of the kernel and the size of the individual particle tending to zero [38,50]; and, the kernel blowing up for small sizes [19]. Without some additional assumptions on either the kernel or initial condition, the above assumptions can cause the formation of particles of infinite size. This phenomenon is known as gelation and has been shown to happen in finite time [56]. Since gelation is not the focus of this paper, we will require more regularity on our coagulation kernel.

Most studies of coagulation-fragmentation equations focus on the case of binary fragmentation; in other words, when particles only fragment into two smaller units (see [45] and the references therein, as well as the previously mentioned works). Although the initial work [48] considers the more general case of multiple fragmentation, where particles can fragment into more than 2 smaller particles, it is difficult to find many results concerning this case. In the setting of density-based equations, the authors of $[47,48]$ work with only an assumption of bounded kernels for both coagulation and fragmentation. Meanwhile, the work [40] allows for linear growth in the rate of fragmentation, but requires a bound on the coagulation kernel. The case where both the coagulation and fragmentation kernels are unbounded is studied in $[28,29]$. 
In this work we will extend the formulation of model (1.2) to the space of Radon measures. This extension allows the unification of the discrete model (1.1) and the continuous (density) model (1.2) under the same framework. In recent years, the space of Radon measures equipped with the bounded Lipschitz norm has been used in the study of population dynamics $[17,18,31,35]$. While many population models have been studied intensely in this setting, the study of coagulation-fragmentation equations in this space is sparse. Mild measure solutions to a coagulation-diffusion equation have been obtained in [50], where state-space of study was the space of finite measures with absolutely continuous first marginal. More so, the model considered does not include any biological processes (i.e. growth, birth, or death). Existence of solutions to a coagulation-fragmentation equation is obtained in [23] via probabilistic means. However, authors in [23] only prove existence of a measure solution in the topology of weak convergence and also do not consider any biological processes. The authors in [20] consider a growth-fragmentation equation with a multiple fragmentation kernel identical to that studied in [28]. They cite well-posedness of their model as a consequence of [18] and do not consider a coagulation term. In this paper, we adopt similar assumptions on our model ingredients as in [20], but will prove well-posedness using a fixed-point approach presented in [6]. Finally, for a structured model without coagulation or fragmentation, [35] proves that solutions are absolutely continuous to the left of the zero characteristic curve. Under similar assumptions, we will extend this result to structured coagulation-fragmentation equations.

The layout of the paper is as follows. In Section 2, we present notation used throughout the paper. In Section 3, we formulate the model, prove some useful properties of the model ingredients, and show the model is indeed well-posed. In Section 4, we analyze the interplay between the biological processes (growth, death and birth) and the physical processes (coagulation and fragmentation). In particular, we study their effects on the regularity of solutions to the structured model. In Section 5, we show that the density equation (1.2) and discrete equations (1.1) are indeed special cases of our model. In Section 6, we present a semidiscrete numerical scheme which we test against a few examples providing approximate error in the BL-norm and the numerical order. Finally, in Section 7 we will provide discussion of the results and some concluding remarks.

\section{PREliminaries AND NOtation}

In this section, we will provide some preliminary notation. The space of finite Radon measures over $\mathbb{R}^{+}:=$ $[0, \infty)$ is denoted by $\mathcal{M}\left(\mathbb{R}^{+}\right)$. The non-negative cone of $\mathcal{M}\left(\mathbb{R}^{+}\right)$will be denoted $\mathcal{M}^{+}\left(\mathbb{R}^{+}\right)$. Unless otherwise stated, both of these spaces will always be equipped with the Bounded-Lipschitz norm given by

$$
\|\mu\|_{\mathrm{BL}}:=\sup _{\|\phi\|_{W^{1, \infty}} \leq 1}\left\{\int_{\mathbb{R}^{+}} \phi(x) \mu(\mathrm{d} x): \phi \in W^{1, \infty}\left(\mathbb{R}^{+}\right)\right\} .
$$

Here, $W^{1, \infty}\left(\mathbb{R}^{+}\right)$is the usual Sobolev space over $\mathbb{R}^{+}$with codomain $\mathbb{R}$ equipped with the usual norm $\|\phi\|_{W^{1, \infty}}:=$ $\|\phi\|_{\infty}+\left\|\phi^{\prime}\right\|_{\infty}$. In the literature, the BL-norm has had a few names such as the flat norm [25,26], the Dudley norm $[22,24]$, and the Fortet-Mourier norm [27,41]. Another norm commonly associated with measures is the total variation norm given by

$$
\|\nu\|_{\mathrm{TV}}=|\nu|\left(\mathbb{R}^{+}\right)=\sup _{\|f\|_{\infty} \leq 1}\left\{\int_{\mathbb{R}^{+}} f \mathrm{~d} \nu: f \in C_{c}\left(\mathbb{R}^{+}\right)\right\} .
$$

It should be noted that while over nonnegative measures they are equivalent, the BL-norm and TV-norm are different on the space of signed measures. In particular, for $\mu \in \mathcal{M}(\mathbb{R})$

$$
\|\mu\|_{\mathrm{BL}} \leq\|\mu\|_{\mathrm{TV}} .
$$

We refer the reader to [32] and the references therein for more information.

We say a sequence $\left(\mu_{n}\right)$ of Radon measures is tight if

$$
\lim _{x \longrightarrow \infty} \sup _{n} \mu_{n}([x, \infty))=0 .
$$


In $\mathcal{M}^{+}\left(\mathbb{R}^{+}\right)$, we additionally have that the BL-norm metrizes weak convergence. That is $\left(\mu_{n}\right)$ converges weakly to $\mu \in \mathcal{M}^{+}\left(\mathbb{R}^{+}\right)$if for every $f \in C_{b}\left(\mathbb{R}^{+}\right)$,

$$
\int_{\mathbb{R}^{+}} f \mathrm{~d}\left(\mu_{n}-\mu\right) \longrightarrow 0
$$

as $n \longrightarrow \infty$. For more detail, see [30,31].

It is often convenient to use the operator notation in place of integration. That is for a function $f$, we say

$$
(\mu, f):=\int_{\mathbb{R}^{+}} f(x) \mu(\mathrm{d} x) .
$$

Finally, we say the flow of a Lipschitz vector field $g(t, x)$ is a function $T_{s, t}^{g}(x)$ which satisfies

$$
\frac{\mathrm{d}}{\mathrm{d} t} T_{s, t}^{g}(x)=g\left(t, T_{s, t}(x)\right), \quad T_{s, s}^{g}(x)=x .
$$

\section{Structured COAGUlation-Fragmentation Equation}

In this section, we formulate a structured coagulation-fragmentation population model on the space of Radon measures. We then establish existence and uniqueness of solutions for the structured coagulation-fragmentation model. Finally, we prove a stability result which will be useful in later sections.

\subsection{Formulation of the model on the space of Radon measures}

The coagulation term we propose is the measure given by

$$
\begin{aligned}
K[\mu](\cdot) & =\frac{1}{2} \int_{\mathbb{R}^{+}} \int_{\mathbb{R}^{+}} \kappa\left(y, y^{\prime}\right) \delta_{y+y^{\prime}}(\cdot) \mu\left(\mathrm{d} y^{\prime}\right) \mu(\mathrm{d} y)-\int_{\mathbb{R}^{+}} \kappa(y, \cdot) \mu(\mathrm{d} y) \mu \\
& =: K^{+}[\mu]-K^{-}[\mu],
\end{aligned}
$$

where $\kappa(x, y)$ represents the rate at which individuals of size $x$ coalesce with individuals of size $y$. The first term in $(3.1), K^{+}$, represents the inflow of individuals due to coagulation. The second term in (3.1), $K^{-}$represents the number of individuals lost due to coagulation. Notice that $K^{ \pm}[\mu]$ are measures which can be described in a distribution sense by

$$
\left(K^{+}[\mu], \phi\right)=\frac{1}{2} \int_{\mathbb{R}^{+}} \int_{\mathbb{R}^{+}} \kappa(y, x) \phi(x+y) \mu(\mathrm{d} x) \mu(\mathrm{d} y)
$$

and

$$
\left(K^{-}[\mu], \phi\right)=\int_{\mathbb{R}^{+}} \int_{\mathbb{R}^{+}} \kappa(y, x) \phi(x) \mu(\mathrm{d} y) \mu(\mathrm{d} x) .
$$

We claim these terms are generalizations of the coagulation terms of the continuous coagulation equation (1.2) given by

$$
K^{+}(u)(x)=\frac{1}{2} \int_{0}^{x} \kappa(y, x-y) u(x-y) u(y) \mathrm{d} y, \quad K^{-}(u)(x)=u(x) \int_{0}^{\infty} \kappa(y, x) u(y) \mathrm{d} y .
$$

Indeed, multiplying $K^{+}(u)$ by a test function $\phi$ and integrating we see that

$$
\begin{aligned}
\frac{1}{2} \int_{0}^{\infty} \int_{0}^{x} \kappa(y, x-y) u(x-y) u(y) \mathrm{d} y \phi(x) \mathrm{d} x & =\frac{1}{2} \int_{0}^{\infty} \int_{y}^{\infty} \kappa(y, x-y) \phi(x) u(x-y) \mathrm{d} x u(y) \mathrm{d} y \\
& =\frac{1}{2} \int_{0}^{\infty} \int_{0}^{\infty} \kappa(y, x) \phi(x+y) u(x) \mathrm{d} x u(y) \mathrm{d} y .
\end{aligned}
$$


which is $\left(K^{+}[\mu], \phi\right)$ for $\mu=u(y) \mathrm{d} y$. An analogous reasoning yields $K^{-}$. Notice that if $\kappa$ is symmetric, i.e. $\kappa(x, y)=\kappa(y, x)$, then

$$
(K[\mu], \phi)=\frac{1}{2} \int_{\mathbb{R}^{+}} \int_{\mathbb{R}^{+}} \kappa(y, x)[\phi(x+y)-\phi(x)-\phi(y)] \mu(\mathrm{d} x) \mu(\mathrm{d} y) .
$$

Notice by formally taking $\mu=\sum_{i \in \mathbb{N}} m_{i} \delta_{x_{i}}$ we can arrive at the discrete coagulation terms given in (1.1) from the traditional Smoluchowski equations [55].

The fragmentation term we propose is given by

$$
F[\mu](\cdot)=\int_{\mathbb{R}^{+}} b(y, \cdot) a(y) \mu(\mathrm{d} y)-a \mu=: F^{+}[\mu]-F^{-}[\mu] .
$$

Here, $a(y)$ represents the global fragmentation rate of individuals of size $y$ and $b(y, \cdot)$ is a measure supported on $[0, y]$ such that $b(y, A)$ represents the probability a particle of size $y$ fragments to a particle with size in the Borel set $A$. The positive term, $F^{+}$, represents the inflow of individuals due to fragmentation, and the negative term, $F^{-}$, represents the number of individuals lost due to fragmentation. Similar to the coagulation terms, $F^{ \pm}[\mu]$ are measures given explicitly by

$$
\left(F^{+}[\mu], \phi\right)=\int_{\mathbb{R}^{+}}(b(y, \cdot), \phi) a(y) \mu(\mathrm{d} y),
$$

where $(b(y, \cdot), \phi)=\int_{0}^{y} \phi(x) b(y, \mathrm{~d} x)$, and

$$
\left(F^{-}[\mu], \phi\right)=\int_{\mathbb{R}^{+}} a(y) \phi(y) \mu(\mathrm{d} y) .
$$

As before these terms are a generalization of the multiple fragmentation terms from (1.2):

$$
F^{+}(u)(x)=\int_{x}^{\infty} b(y, x) a(y) u(y) \mathrm{d} y, \quad F^{-}(u)(x)=a(x) u(x) .
$$

Here, following [23], we allow $b(y, \cdot)=b(y, \mathrm{~d} x)$ to be a non-negative measure supported in $[0, y]$.

With these generalized terms, we propose the following model:

$$
\left\{\begin{array}{lr}
\partial_{t} \mu+\partial_{x}(g(t, \mu) \mu)+d(t, \mu) \mu=K[\mu]+F[\mu], & (t, x) \in(0, T) \times(0, \infty) \\
g(t, \mu)(0) D_{\mathrm{d} x} \mu(0)=\int_{\mathbb{R}^{+}} \beta(t, \mu)(y) \mu(\mathrm{d} y), & t \in[0, T] \\
\mu(0)=\mu_{0} \in \mathcal{M}^{+}\left(\mathbb{R}^{+}\right) &
\end{array}\right.
$$

where

$$
\begin{aligned}
\mu & :[0, T] \longrightarrow \mathcal{M}^{+}\left(\mathbb{R}^{+}\right), \\
g, d, \beta & :[0, T] \times \mathcal{M}^{+}\left(\mathbb{R}^{+}\right) \longrightarrow W^{1, \infty}\left(\mathbb{R}^{+}\right), \\
K & : \mathcal{M}^{+}\left(\mathbb{R}^{+}\right) \longrightarrow \mathcal{M}\left(\mathbb{R}^{+}\right), \\
F & : \mathcal{M}^{+}\left(\mathbb{R}^{+}\right) \longrightarrow \mathcal{M}\left(\mathbb{R}^{+}\right) .
\end{aligned}
$$

As in the density model (1.2), the functions $g, d$, and $\beta$ are nonnegative and represent the growth, death, and birth functions, respectively. They are assumed to be influenced by both time, $t$, and the solution to the population model, $\mu(t)$. In applications (e.g. see $[2,8,18,21]$ ), it is common to choose $\beta, g$ and $d$ to depend on a weighted mean of the population in the following form:

$$
\beta(t, \mu)(x)=B\left(t, x, \int_{\mathbb{R}^{+}} K_{B}(y) \mathrm{d} \mu(y)\right)
$$


and similar expressions for $g$ and $d$, for given maps $B:[0, T] \times \mathbb{R}^{+} \times \mathbb{R}^{+} \rightarrow \mathbb{R}^{+}$and $K_{B}: \mathbb{R}^{+} \rightarrow \mathbb{R}^{+}$. Common physically motivated model functions utilize BevertonHolt type [12] or Ricker type [52] nonlinearities with respect to the weighted mean of the population and of a Von Bertalanffy type [51] model with respect to structure $x$. In the boundary condition, $D_{\mathrm{d} x} \mu(x)$ represents the Radon-Nikodym derivative of $\mu$ with respect to the Lebesgue measure, $\mathrm{d} x$, evaluated at $x$.

We impose the following assumptions on the growth, death and birth functions:

(A1) For any $R>0$, there exists $L_{R}>0$ such that for all $\left\|\mu_{i}\right\|_{\mathrm{TV}} \leq R$ and $t_{i} \in[0, \infty)(i=1,2)$ the following hold

$$
\begin{aligned}
\left\|g\left(t_{1}, \mu_{1}\right)-g\left(t_{2}, \mu_{2}\right)\right\|_{\infty} & \leq L_{R}\left(\left|t_{1}-t_{2}\right|+\left\|\mu_{1}-\mu_{2}\right\|_{\mathrm{BL}}\right), \\
\left\|d\left(t_{1}, \mu_{1}\right)-d\left(t_{2}, \mu_{2}\right)\right\|_{\infty} & \leq L_{R}\left(\left|t_{1}-t_{2}\right|+\left\|\mu_{1}-\mu_{2}\right\|_{\mathrm{BL}}\right), \\
\left\|\beta\left(t_{1}, \mu_{1}\right)-\beta\left(t_{2}, \mu_{2}\right)\right\|_{\infty} & \leq L_{R}\left(\left|t_{1}-t_{2}\right|+\left\|\mu_{1}-\mu_{2}\right\|_{\mathrm{BL}}\right) .
\end{aligned}
$$

(A2) There exists $\zeta>0$ such that for all $T>0$

$$
\sup _{t \in[0, T]} \sup _{\mu \in \mathcal{M}^{+}\left(\mathbb{R}^{+}\right)}\|g(t, \mu)\|_{W^{1, \infty}}+\|d(t, \mu)\|_{W^{1, \infty}}+\|\beta(t, \mu)\|_{W^{1, \infty}}<\zeta .
$$

(A3) For all $(t, \mu) \in[0, \infty) \times \mathcal{M}^{+}\left(\mathbb{R}^{+}\right)$,

$$
g(t, \mu)(0)>0 .
$$

We assume that the coagulation kernel $\kappa$ satisfies the following assumption:

(K) $\kappa$ is symmetric, nonnegative, bounded by a constant $C_{\kappa}$, and globally Lipschitz with Lipschitz constant $L_{\kappa}$.

We assume that the fragmentation kernel satisfies the following assumptions:

(F1) $a \in W^{1, \infty}\left(\mathbb{R}^{+}\right)$is non-negative,

(F2) for any $y \geq 0, b(y, \mathrm{~d} x)$ is a measure such that

(i) $b(y, \mathrm{~d} x)$ is non-negative and supported in $[0, y]$ so that for all $y>0$ there exist a $C_{b}>0$ such that $b\left(y, \mathbb{R}^{+}\right)<C_{b}$,

(ii) there exists $L_{b}$ such that

$$
\|b(y, \cdot)-b(\bar{y}, \cdot)\|_{\mathrm{BL}} \leq L_{b}|y-\bar{y}|
$$

(iii) $(b(y, \cdot), x)=y$.

It follows from (F2) that for any $\phi$, with $\|\phi\|_{W^{1, \infty}} \leq 1$, the function $\Phi[\phi](y)=(b(y, \cdot), \phi)$ is bounded Lipschitz with $\|\Phi[\phi](y)\|_{W^{1, \infty}} \leq \bar{C}_{b}=\max \left\{C_{b}, L_{b}\right\}$.

Given $T \geq 0$, we say a function $\mu \in C\left([0, T], \mathcal{M}^{+}\left(\mathbb{R}^{+}\right)\right)$is a weak solution to (3.8) if for all $\phi \in\left(C^{1} \cap\right.$ $\left.W^{1, \infty}\right)\left([0, T] \times \mathbb{R}^{+}\right)$, and for all $t \in[0, T]$ the following holds:

$$
\begin{aligned}
\int_{\mathbb{R}^{+}} \phi(t, x) \mu_{t}(\mathrm{~d} x)-\int_{\mathbb{R}^{+}} \phi(0, x) \mu_{0}(\mathrm{~d} x)= & \int_{0}^{t} \int_{\mathbb{R}^{+}}\left[\partial_{t} \phi(s, x)+g\left(s, \mu_{s}\right)(x) \partial_{x} \phi(s, x)-d\left(s, \mu_{s}\right)(x) \phi(s, x)\right] \mu_{s}(\mathrm{~d} x) \mathrm{d} s \\
& +\int_{0}^{t}\left(K\left[\mu_{s}\right]+F\left[\mu_{s}\right], \phi(s, \cdot)\right) \mathrm{d} s+\int_{0}^{t} \int_{\mathbb{R}^{+}} \phi(s, 0) \beta\left(s, \mu_{s}\right)(x) \mu_{s}(\mathrm{~d} x) \mathrm{d} s .
\end{aligned}
$$

Notice that we can also write model (3.8) with the boundary condition as a source term:

$$
\partial_{t} \mu+\partial_{x}(g(t, \mu) \mu)+d(t, \mu) \mu=K[\mu]+F[\mu]+S(t)\left[\mu_{t}\right]
$$

where $S(t)[\mu]=\left(\int_{0}^{\infty} \beta(t, \mu)(y) \mu(\mathrm{d} y)\right) \delta_{x=0}$. 


\subsection{Well-posedness of the structured coagulation-fragmentation equation}

Here, we aim to prove model (3.8) is well-posed. To this end, we present the following propositions which describe useful properties of the source terms.

Proposition 3.1. For every $\mu \in \mathcal{M}\left(\mathbb{R}^{+}\right)$we have

$$
\|K[\mu]\|_{\mathrm{TV}} \leq \frac{3}{2} C_{\kappa}\|\mu\|_{\mathrm{TV}}^{2} .
$$

For every $\mu, \nu \in \mathcal{M}\left(\mathbb{R}^{+}\right)$with $\|\mu\|_{\mathrm{TV}},\|\nu\|_{\mathrm{TV}} \leq R$,

$$
\|K[\mu]-K[\nu]\|_{\mathrm{BL}} \leq \bar{L}_{\kappa, R}\|\mu-\nu\|_{\mathrm{BL}},
$$

where $\bar{L}_{\kappa, R}$ is a constant depending only on $C_{\kappa}, L_{\kappa}$, and $R$.

Proof. To prove (3.12) notice that

$$
\left\|K^{+}[\mu]\right\|_{\mathrm{TV}} \leq \frac{1}{2} \int_{\mathbb{R}^{+}} \int_{\mathbb{R}^{+}} \kappa(x, y)|\mu|(\mathrm{d} x)|\mu|(\mathrm{d} y) \leq \frac{1}{2} C_{\kappa}\|\mu\|_{\mathrm{TV}}^{2}
$$

and also

$$
\left\|K^{-}[\mu]\right\|_{\mathrm{TV}} \leq \int_{\mathbb{R}^{+}} \int_{\mathbb{R}^{+}} \kappa(x, y)|\mu|(\mathrm{d} x)|\mu|(\mathrm{d} y) \leq C_{\kappa}\|\mu\|_{\mathrm{TV}}^{2} .
$$

Since $\|K[\mu]\|_{\mathrm{TV}}=\left\|K^{+}[\mu]-K^{-}[\mu]\right\|_{\mathrm{TV}} \leq\left\|K^{+}[\mu]\right\|_{\mathrm{TV}}+\left\|K^{-}[\mu]\right\|_{\mathrm{TV}}$, we obtain (3.12).

To prove (3.13), let $\phi \in W^{1, \infty}\left(\mathbb{R}^{+}\right)$be such that $\|\phi\|_{W^{1, \infty}} \leq 1$. Then

$$
\begin{aligned}
2\left|\left(K^{+}[\mu]-K^{+}[\nu], \phi\right)\right|= & \left|\int_{\mathbb{R}^{+}} \int_{\mathbb{R}^{+}} \kappa\left(y, y^{\prime}\right) \phi\left(y+y^{\prime}\right) \mu(\mathrm{d} y) \mu\left(\mathrm{d} y^{\prime}\right)-\int_{\mathbb{R}^{+}} \int_{\mathbb{R}^{+}} \kappa\left(y, y^{\prime}\right) \phi\left(y+y^{\prime}\right) \nu(\mathrm{d} y) \nu\left(\mathrm{d} y^{\prime}\right)\right| \\
= & \mid \int_{\mathbb{R}^{+}} \int_{\mathbb{R}^{+}} \kappa\left(y, y^{\prime}\right) \phi\left(y+y^{\prime}\right) \mu(\mathrm{d} y)(\mu-\nu)\left(\mathrm{d} y^{\prime}\right) \\
& +\int_{\mathbb{R}^{+}} \int_{\mathbb{R}^{+}} \kappa\left(y, y^{\prime}\right) \phi\left(y+y^{\prime}\right) \nu\left(\mathrm{d} y^{\prime}\right)(\mu-\nu)(\mathrm{d} y) \mid .
\end{aligned}
$$

Since $\kappa$ is symmetric,

$$
\begin{aligned}
2\left|\left(K^{+}[\mu]-K^{+}[\nu], \phi\right)\right| & =\left|\int_{\mathbb{R}^{+}} \int_{\mathbb{R}^{+}} \kappa\left(y, y^{\prime}\right) \phi\left(y+y^{\prime}\right)(\mu-\nu)(\mathrm{d} y)(\mu+\nu)\left(\mathrm{d} y^{\prime}\right)\right| \\
& \leq \int_{\mathbb{R}^{+}}\left|\int_{\mathbb{R}^{+}} \kappa\left(y, y^{\prime}\right) \phi\left(y+y^{\prime}\right)(\mu-\nu)(\mathrm{d} y)\right|(|\mu|+|\nu|)\left(\mathrm{d} y^{\prime}\right) .
\end{aligned}
$$

For a given $y^{\prime} \geq 0$, the function $y \mapsto \kappa\left(y, y^{\prime}\right) \phi\left(y+y^{\prime}\right)$ is bounded Lipschitz with norm $\leq C_{\kappa}+L_{\kappa}$. Thus

$$
2\left|\left(K^{+}[\mu]-K^{+}[\nu], \phi\right)\right| \leq\left(C_{\kappa}+L_{\kappa}\right)\left(\|\mu\|_{\mathrm{TV}}+\|\nu\|_{\mathrm{TV}}\right)\|\mu-\nu\|_{\mathrm{BL}} .
$$

Taking the supremum over all such $\phi$ gives

$$
\left\|K^{+}[\mu]-K^{+}[\nu]\right\|_{\mathrm{BL}} \leq \frac{1}{2}\left(C_{\kappa}+L_{\kappa}\right)\left(\|\mu\|_{\mathrm{TV}}+\|\nu\|_{\mathrm{TV}}\right)\|\mu-\nu\|_{\mathrm{BL}}
$$

In the same way

$$
\left|\left(K^{-}[\mu]-K^{-}[\nu], \phi\right)\right|=\left|\int_{\mathbb{R}^{+}} \int_{\mathbb{R}^{+}} \kappa(y, x) \phi(x) \mu(\mathrm{d} y) \mu(\mathrm{d} x)-\int_{\mathbb{R}^{+}} \int_{\mathbb{R}^{+}} \kappa(y, x) \phi(x) \nu(\mathrm{d} y) \nu(\mathrm{d} x)\right|
$$




$$
\begin{aligned}
& =\left|\int_{\mathbb{R}^{+}} \int_{\mathbb{R}^{+}} \kappa(y, x) \phi(x) \mu(\mathrm{d} y)(\mu-\nu)(\mathrm{d} x)+\int_{\mathbb{R}^{+}} \int_{\mathbb{R}^{+}} \kappa(y, x) \phi(x)(\mu-\nu)(\mathrm{d} y) \nu(\mathrm{d} x)\right| \\
& \leq \int_{\mathbb{R}^{+}}\left|\int_{\mathbb{R}^{+}} \kappa(y, x) \phi(x)(\mu-\nu)(\mathrm{d} x)\right||\mu|(\mathrm{d} y)+\int_{\mathbb{R}^{+}}\left|\int_{\mathbb{R}^{+}} \kappa(y, x)(\mu-\nu)(\mathrm{d} y)\right||\phi(x) \| \nu|(\mathrm{d} x) \\
& \leq\left(\left(L_{\kappa}+C_{\kappa}\right)\|\mu\|_{\mathrm{TV}}+\|\nu\|_{\mathrm{TV}} \max \left\{L_{\kappa}, C_{\kappa}\right\}\right)\|\mu-\nu\|_{\mathrm{BL}}
\end{aligned}
$$

Combining these two results we see that

$$
\|K[\mu]-K[\nu]\|_{\mathrm{BL}} \leq \bar{L}_{K, R}\|\mu-\nu\|_{\mathrm{BL}} .
$$

Next we have the following proposition concerning the fragmentation term:

Proposition 3.2. For any $\mu \in \mathcal{M}\left(\mathbb{R}^{+}\right)$we have

$$
\|F[\mu]\|_{\mathrm{TV}} \leq\left(\bar{C}_{b}+1\right)\|a\|_{\infty}\|\mu\|_{\mathrm{TV}} .
$$

and

$$
\|F[\mu]-F[\nu]\|_{\mathrm{BL}} \leq C_{a, b}\|\mu-\nu\|_{\mathrm{BL}}
$$

Proof. Clearly,

$$
\left\|F^{-}[\mu]\right\|_{\mathrm{TV}} \leq\|a\|_{\infty}\|\mu\|_{\mathrm{TV}}
$$

and

$$
\left\|F^{-}[\mu]-F^{-}[\nu]\right\|_{\mathrm{BL}} \leq\|a\|_{W^{1, \infty}}\|\mu-\nu\|_{\mathrm{BL}}=C_{a}\|\mu-\nu\|_{\mathrm{BL}} .
$$

Also,

$$
\left\|F^{+}[\mu]\right\|_{\mathrm{TV}} \leq\|a\|_{\infty}\|\mu\|_{\mathrm{TV}}\|\Phi(1)\|_{\infty}=\bar{C}_{b}\|a\|_{\infty}\|\mu\|_{\mathrm{TV}}
$$

and

$$
\left\|F^{+}[\mu]-F^{+}[\nu]\right\|_{\mathrm{BL}} \leq\|\mu-\nu\|_{\mathrm{BL}} \sup _{\|\phi\|_{W^{1, \infty}} \leq 1}\|\Phi[\phi] a\|_{W^{1, \infty}}=C_{a, b}\|\mu-\nu\|_{\mathrm{BL}}
$$

The following proposition is immediate from assumptions (A1) and (A2).

Proposition 3.3. $S(t)[\mu]$ satisfies the following:

- $S(t)[\mu] \geq 0$ whenever $\mu \geq 0$;

- $\|S(t)[\mu]\|_{\mathrm{TV}} \leq \zeta\|\mu\|_{\mathrm{TV}}$

- For any $t \geq 0$ and for any $\mu, \nu$ with $\|\mu\|_{\mathrm{TV}},\|\nu\|_{\mathrm{TV}} \leq R$,

$$
\|S(t)[\mu]-S(t)[\nu]\|_{\mathrm{BL}} \leq\left(\zeta+R L_{R}\right)\|\mu-\nu\|_{\mathrm{BL}} .
$$

We are now ready to show model (3.8) is well posed. More precisely, we have the following result:

Theorem 3.4. Assume that assumptions (A1), (A2), (A3), (K), (F1), and (F2) hold. Given an initial condition $\mu_{0} \in \mathcal{M}^{+}\left(\mathbb{R}^{+}\right)$, there exists a unique global solution $\mu \in C\left([0, \infty), \mathcal{M}^{+}\left(\mathbb{R}^{+}\right)\right)$of equation (3.8). Moreover, if $\mu_{0}$ has finite total mass in the sense that $\int_{\mathbb{R}^{+}} x \mu_{0}(\mathrm{~d} x)<\infty$, then for any $T \geq 0$ there exists $C_{T}>0$ such that

$$
\int_{\mathbb{R}^{+}} x \mu_{t}(\mathrm{~d} x) \leq C_{T} \quad t \in[0, T] .
$$

In particular, if $g=d=\beta=0$ then mass is conserved in the sense that $\int_{\mathbb{R}^{+}} x \mu_{t}(\mathrm{~d} x)=\int_{\mathbb{R}^{+}} x \mu_{0}(\mathrm{~d} x)$ for any $t \geq 0$. 
Proof. Let

$$
B(t, \mu):=F^{+}[\mu]+K^{+}[\mu]+S(t)[\mu]
$$

and

$$
\bar{N}(t, x, \mu):=-d(t, \mu)(x)-a(x)-\int_{\mathbb{R}^{+}} \kappa(y, x) \mu(\mathrm{d} y) .
$$

Then equation (3.8) reads

$$
\partial_{t} \mu+\partial_{x}(g(t, \mu) \mu)=B(t, \mu)+\bar{N}(t, \cdot, \mu) \mu .
$$

For any $R>0$, denote $\mathcal{M}_{R}(\mathbb{R}):=\left\{\mu \in \mathcal{M}(\mathbb{R}):\|\mu\|_{\mathrm{TV}} \leq R\right\}$. Notice $\mathcal{M}_{R}(\mathbb{R})$ is complete for the BL norm. According to Propositions 3.1-3.3, B: $\mathbb{R}^{+} \times \mathcal{M}(\mathbb{R}) \rightarrow \mathcal{M}(\mathbb{R})$ and $\bar{N}: \mathbb{R}^{+} \times \mathbb{R} \times \mathcal{M}(\mathbb{R}) \rightarrow W^{1, \infty}(\mathbb{R})$ are continuous and satisfy the following properties:

(B1) $B[t, \mu] \in \mathcal{M}^{+}(R)$ for any $t \geq 0$ if $\mu \in \mathcal{M}^{+}(\mathbb{R})$,

(B2) for any $R>0$ there exists $C_{B, R}>0$ and $L_{B, R}>0$ such that for any $t \geq 0$ and any $\mu, \tilde{\mu} \in \mathcal{M}_{R}\left(\mathbb{R}^{d}\right)$,

$$
\|B(t, \mu)\|_{\mathrm{TV}} \leq C_{B, R}, \quad \text { and } \quad\|B(t, \mu)-B(t, \tilde{\mu})\|_{\mathrm{BL}} \leq L_{B, R}\|\mu-\tilde{\mu}\|_{\mathrm{BL}},
$$

(N1) for any $R>0$, there exist $L_{\bar{N}, R}>0$ and $C_{\bar{N}, R}>0$ such that for any $t \geq 0, x \in \mathbb{R}$, and any $\mu, \tilde{\mu} \in \mathcal{M}_{R}(\mathbb{R})$,

$$
\|\bar{N}(t, \cdot, \mu)\|_{W^{1, \infty}} \leq C_{\bar{N}, R} \quad \text { and } \quad|\bar{N}(t, x, \mu)-\bar{N}(t, x, \tilde{\mu})| \leq L_{\bar{N}, R}\|\mu-\tilde{\mu}\|_{\mathrm{BL}} .
$$

It follows from standard arguments (e.g. $[5,6]$ and references therein) that equation (3.8) has a unique solution $\mu \in C\left(\left[0, T^{*}\right), \mathcal{M}\left(\mathbb{R}^{+}\right)\right)$which is nonnegative and defined on a maximal time interval $\left[0, T^{*}\right)$. Moreover, $T^{*}<\infty$ if and only if $\lim _{t \rightarrow T^{*}}\left\|\mu_{t}\right\|_{\mathrm{TV}}=\infty$. Indeed this follows applying Banach fixed-point Theorem to the map $\Gamma: X_{T} \rightarrow X_{T}$ with

$$
X_{T}=\left\{\mu \in C\left([0, T], \mathcal{M}\left(\mathbb{R}^{+}\right)\right): \mu(0)=\mu_{0},\|\mu\|_{\mathrm{TV}} \leq 2\left\|\mu_{0}\right\|_{\mathrm{TV}} \forall t \in[0, T]\right\},
$$

and

$$
\Gamma[\mu]_{t}=T_{0, t}^{g} \sharp \mu_{0}+\int_{0}^{t} T_{s, t}^{g} \sharp N(s, \mu) \mathrm{d} s,
$$

where $N(s, \mu):=\bar{N}(s, \cdot, \mu) \mu+B(s, \mu), T_{s, t}^{g}$ is the flow of the vector field $(t, x) \rightarrow g\left(t, \mu_{t}\right)(x)$, and the measure $f \sharp \mu$ denotes the pushforward of $\mu$ through $f$. We can then prove that taking $T$ small enough, $\Gamma\left(X_{T}\right) \subset X_{T}$ and $\Gamma$ is a strict contraction. We then deduce that (3.8) has a unique solution $\mu \in C\left(\left[0, T^{*}\right), \mathcal{M}\left(\mathbb{R}^{+}\right)\right)$. If moreover $\mu_{0} \geq 0$ we can then prove as ([6], Prop. 5.1 and Thm 5.2) that $\mu_{t} \geq 0$ for any $t<T^{*}$.

Recall that if $T^{*}<\infty$ then it must be $\lim _{t \rightarrow T^{*}}\left\|\mu_{t}\right\|_{\mathrm{TV}}=\infty$. Thus to prove that $T^{*}=\infty$, it is enough to verify that there exists $C>0$ such that

$$
\left\|\mu_{t}\right\|_{\mathrm{TV}} \leq\left\|\mu_{0}\right\|_{\mathrm{TV}} \exp (C t) \quad \text { for any } t \in\left[0, T^{*}\right) .
$$

To begin, we first note for any finite non-negative measure $\mu$,

$$
(K[\mu], 1)=-\frac{1}{2} \int_{\mathbb{R}^{+}} \int_{\mathbb{R}^{+}} \kappa(x, y) \mu(\mathrm{d} x) \mu(\mathrm{d} y) \leq 0
$$

and

$$
(F[\mu], 1)=\int_{\mathbb{R}^{+}}(b(y, \cdot), 1) a(y) \mu(\mathrm{d} y)-\int_{\mathbb{R}^{+}} a(y) \mu(\mathrm{d} y) \leq \int_{\mathbb{R}^{+}}\left(C_{b}-1\right) a(y) \mu(\mathrm{d} y) .
$$

Therefore, taking $\phi(t, x) \equiv 1$ in (3.10), we can arrive at

$$
\begin{aligned}
\left(\mu_{t}, 1\right) & \leq\left(\mu_{0}, 1\right)+\int_{0}^{t} \int_{\mathbb{R}^{+}}\left[\left(C_{b}-1\right) a(y)+\beta\left(s, \mu_{s}\right)(y)\right] \mu_{s}(\mathrm{~d} y) \mathrm{d} s \\
& \leq\left(\mu_{0}, 1\right)+\left[\left(C_{b}-1\right)\|a\|_{\infty}+\zeta\right] \int_{0}^{t}\left(\mu_{s}, 1\right) \mathrm{d} s
\end{aligned}
$$


The Gronwall inequality then gives (3.18) with $C=\left(C_{b}-1\right)\|a\|_{\infty}+\zeta$.

Now, assume that $\int_{0}^{\infty} x \mu_{0}(\mathrm{~d} x)<\infty$. Let $R>0$ and consider a smooth regularization of the test function $\phi_{R}(x)=\min \{x, R\}$ in the weak formulation (3.10). Since $\phi_{R}(x+y)-\phi_{R}(x)-\phi_{R}(y) \leq 0$ for any $x, y \geq 0$, we have from equation (3.5) that $\left(K\left[\mu_{t}\right], \phi_{R}\right) \leq 0$. Moreover, $\phi_{R}(0)=0$ and $\phi_{R} \geq 0$. We thus obtain

$$
\left(\mu_{t}, \phi_{R}\right) \leq\left(\mu_{0}, \phi_{R}\right)+\int_{0}^{t} \int_{\mathbb{R}^{+}} g\left(s, \mu_{s}\right)(y) \phi_{R}^{\prime}(y) \mu_{s}(\mathrm{~d} y) \mathrm{d} s+\int_{0}^{t} \int_{\mathbb{R}^{+}}\left(b(y, \cdot), \phi_{R}\right) a(y) \mu_{s}(\mathrm{~d} y) \mathrm{d} s .
$$

Using (A2) and (3.18), we can bound the 2 nd term on the right-hand side by $C_{T, \zeta}$ for $t \in[0, T]$. Using that $\phi_{R}(x) \leq x,(b(y, \mathrm{~d} x), x)=y$, and $(\mathrm{A} 2)$, we have

$$
\begin{aligned}
\left(\mu_{t}, \phi_{R}\right) & \leq\left(\mu_{0}, x\right)+C_{T, \zeta}+\int_{0}^{t} \int_{\mathbb{R}^{+}} y a(y) \mu_{s}(\mathrm{~d} y) \mathrm{d} s \\
& \leq\left(\mu_{0}, x\right)+C_{T, \zeta}+\|a\|_{\infty} \int_{0}^{t}\left(\mu_{s}, x\right) \mathrm{d} s .
\end{aligned}
$$

Passing to the limit $R \rightarrow \infty$ using the Monotone Convergence Theorem, we deduce

$$
\left(\mu_{t}, x\right) \leq\left(\mu_{0}, x\right)+C_{T, \zeta}+\|a\|_{\infty} \int_{0}^{t}\left(\mu_{s}, x\right) \mathrm{d} s .
$$

The Gronwall inequality then gives

$$
\left(\mu_{t}, x\right) \leq\left(\left(\mu_{0}, x\right)+C_{T, \zeta}\right) e^{\|a\|_{\infty} t} .
$$

As a consequence we can use any continuous test-function $\phi$ with linear growth, i.e. $|\phi(x)| \leq C(1+|x|)$. In particular, we can take $\phi(x)=x$ in equation (3.10). Since $\left(K\left[\mu_{t}\right], x\right)=\left(F\left[\mu_{t}\right], x\right)=0$, we obtain

$$
\left(\mu_{t}, x\right)=\left(\mu_{0}, x\right)+\int_{0}^{t} \int_{\mathbb{R}^{+}} g\left(s, \mu_{s}\right)(y) \mu_{s}(\mathrm{~d} y) \mathrm{d} s-\int_{0}^{t} \int_{\mathbb{R}^{+}} x d\left(s, \mu_{s}\right)(x) \mu_{s}(\mathrm{~d} y) \mathrm{d} s .
$$

In particular, if $g=d=0$, we have $\left(\mu_{t}, x\right)=\left(\mu_{0}, x\right)$ i.e. mass is conserved for any $t \geq 0$.

Remark 3.5. In applications the smallest size will not be of size 0 but rather some $x_{0}>0$. Model (3.11) and the theorem above can be adjusted for such applications by shifting the Dirac measure at 0 to $x_{0}$, requiring $g\left(t, \mu_{t}\right)\left(x_{0}\right)>0$, and requiring $b(y, \cdot)$ to be supported on $\left[x_{0}, y\right)$. In this case, the mass conservation equation would be

$$
\begin{aligned}
\left(\mu_{t}, x\right)= & \left(\mu_{0}, x\right)+\int_{0}^{t} \int_{\mathbb{R}^{+}} g\left(s, \mu_{s}\right)(y) \mu_{s}(\mathrm{~d} y) \mathrm{d} s-\int_{0}^{t} \int_{\mathbb{R}^{+}} x d\left(s, \mu_{s}\right)(x) \mu_{s}(\mathrm{~d} x) \mathrm{d} s \\
& +\int_{0}^{t} \int_{\mathbb{R}^{+}} x_{0} \beta\left(s, \mu_{s}\right)(x) \mu_{s}(\mathrm{~d} x) \mathrm{d} s .
\end{aligned}
$$

\subsection{A stability result}

Let us consider a sequence of equations

$$
\left\{\begin{array}{lr}
\partial_{t} \mu+\partial_{x}\left(g^{n}(t, \mu) \mu\right)+d^{n}(t, \mu) \mu=K^{n}[\mu]+F^{n}[\mu], & (t, x) \in(0, \infty) \times(0, \infty) \\
g^{n}(t, \mu)(0) D_{\mathrm{d} x} \mu(0)=\int_{\mathbb{R}^{+}} \beta^{n}(t, \mu)(y) \mu(\mathrm{d} y), & t \geq 0 \\
\mu^{n}(0) \in \mathcal{M}^{+}\left(\mathbb{R}^{+}\right), \int_{0}^{\infty}(1+x) \mu^{n}(0)(\mathrm{d} x)<\infty &
\end{array}\right.
$$


where

$$
K^{n}[\mu](\cdot)=\frac{1}{2} \int_{\mathbb{R}^{+}} \int_{\mathbb{R}^{+}} \kappa^{n}\left(y, y^{\prime}\right) \delta_{y+y^{\prime}}(\cdot) \mu\left(\mathrm{d} y^{\prime}\right) \mu(\mathrm{d} y)-\int_{\mathbb{R}^{+}} \kappa^{n}(y, \cdot) \mu(\mathrm{d} y) \mu
$$

and

$$
F[\mu](\cdot)=\int_{\mathbb{R}^{+}} b^{n}(y, \cdot) a^{n}(y) \mu(\mathrm{d} y)-a^{n} \mu .
$$

Let us assume that

(S1) the functions $g^{n}, d^{n}, \beta^{n}, \kappa^{n}, a^{n}, b^{n}, n \in \mathbb{N}$, satisfy assumptions (A1), (A2), (A3), (K), (F1), and (F2).

It then follows from Theorem 3.4 that (3.20) has a unique solution $\mu^{n} \in C\left([0, \infty), \mathcal{M}\left(\mathbb{R}_{+}\right)\right.$such that $\int_{0}^{\infty} x \mu^{n}(t)(\mathrm{d} x)<\infty$. Under some additional assumptions on the coefficients of $(3.20)$ we can extract from $\mu^{n}$ a subsequence converging to a solution of (3.8).

Theorem 3.6. Assume that the functions $g^{n}, d^{n}, \beta^{n}, \kappa^{n}, a^{n}, b^{n}, n \in \mathbb{N}$, satisfy assumptions (S1) and also that:

(S2) There exists $C>0$ such that $\left\|\kappa^{n}\right\|_{\infty},\left\|a^{n}\right\|_{\infty} \leq C$ and there exists functions $\kappa$, a such that

$$
\kappa^{n} \rightarrow \kappa, a^{n} \rightarrow a \quad \text { uniformly on compact sets. }
$$

(S3) There exists $C>0$ and a function $b: \mathbb{R}_{+} \rightarrow \mathcal{M}\left(\mathbb{R}_{+}\right)$such that $\left(b^{n}(y), 1\right) \leq C$ for any $y \geq 0$ and $n \in \mathbb{N}$, and for any $\phi \in C_{c}^{\infty}\left(\mathbb{R}^{+}\right)$,

$$
\left(b^{n}(y), \phi\right) \rightarrow(b(y), \phi) \quad \text { uniformly for } y \text { in a compact set. }
$$

(S4) There exist functions $g, d, \beta:[0, \infty) \times \mathcal{M}^{+}\left(\mathbb{R}^{+}\right) \longrightarrow W^{1, \infty}\left(\mathbb{R}^{+}\right)$such that for any $t \geq 0$ and any sequence of measures $m^{n} \in \mathcal{M}^{+}\left(\mathbb{R}^{+}\right)$converging weakly to $m \in \mathcal{M}^{+}\left(\mathbb{R}^{+}\right)$we have

$$
g^{n}\left(t, m^{n}\right) \rightarrow g(t, m), \quad d^{n}\left(t, m^{n}\right) \rightarrow d(t, m), \quad \beta^{n}\left(t, m^{n}\right) \rightarrow \beta(t, m)
$$

uniformly on compact sets of $\mathbb{R}^{+}$.

Concerning the initial condition $\mu^{n}(0) \in \mathcal{M}^{+}\left(\mathbb{R}_{+}\right)$, we assume that $\int_{\mathbb{R}^{+}}(1+x) \mu_{0}^{n}(\mathrm{~d} x) \leq C$ and $\mu_{0}^{n} \longrightarrow \mu_{0}$ in the $B L$ norm for some $\mu_{0} \in \mathcal{M}^{+}\left(\mathbb{R}^{+}\right)$.

Denote $\mu^{n}$ the solution of (3.20). Then, there exists $\mu \in C\left(\mathbb{R}^{+}, \mathcal{M}^{+}\left(\mathbb{R}^{+}\right)\right)$such that, along a subsequence, $\mu^{n} \rightarrow \mu$ in $C\left([0, T], \mathcal{M}^{+}\left(\mathbb{R}^{+}\right)\right)$for any $T>0$, and $\mu$ is a solution of $(3.8)$.

Proof. We have

$$
\left(K\left[\mu^{n}\right], 1\right)=-\frac{1}{2} \int_{0}^{\infty} \int_{0}^{\infty} \kappa^{n}(x, y) \mu_{t}^{n}(\mathrm{~d} x) \mu_{t}^{n}(\mathrm{~d} y) \leq 0
$$

and

$$
\left|\left(F^{n}\left[\mu_{t}^{n}\right], 1\right)\right| \leq \int\left(\left|\left(b^{n}(y), 1\right)\right|+1\right)\left|a^{n}(y)\right| \mathrm{d} \mu_{t}^{n} \leq \sup _{y, n}\left(\left\|a^{n}\right\|_{\infty}+\left|\left(b^{n}(y), 1\right)\right|\right)\left(\mu_{t}^{n}, 1\right)=C\left(\mu_{t}^{n}, 1\right) .
$$

Moreover, $\left(\mu_{0}^{n}, 1\right) \rightarrow\left(\mu_{0}, 1\right)$ so that $\left(\mu_{0}^{n}, 1\right) \leq C$. Taking $\phi=1$ in the weak formulation of (3.20) we thus obtain

$$
\left(\mu_{t}^{n}, 1\right) \leq\left(\mu_{0}^{n}, 1\right)+C \int_{0}^{t}\left(\mu_{s}^{n}, 1\right) \mathrm{d} s \leq C+C \int_{0}^{t}\left(\mu_{s}^{n}, 1\right) \mathrm{d} s .
$$

It then follows from Gronwall inequality that for any $T>0$,

$$
\left(\mu_{t}^{n}, 1\right) \leq C_{T} \quad t \in[0, T]
$$


As in the proof of Theorem 3.4, using $\phi_{R}(x)=\min \{x, R\}, R>0$, as a test-function we obtain

$$
\begin{aligned}
\left(\mu_{t}^{n}, \phi_{R}\right) & \leq\left(\mu_{0}^{n}, \phi_{R}\right)+\int_{0}^{t} \int_{\mathbb{R}^{+}} g^{n}\left(s, \mu_{s}^{n}\right)(y) \phi_{R}^{\prime}(y) \mu_{s}^{n}(\mathrm{~d} y) \mathrm{d} s+\int_{0}^{t} \int_{\mathbb{R}^{+}}\left(b^{n}(y, \cdot), \phi_{R}\right) a^{n}(y) \mu_{s}^{n}(\mathrm{~d} y) \mathrm{d} s \\
& \leq C_{T}+C \int_{0}^{t}\left(\mu_{s}^{n}, x\right) \mathrm{d} s .
\end{aligned}
$$

Letting $R \rightarrow \infty$ using the monotone convergence theorem, and then applying Gronwall inequality we obtain

$$
\left(\mu_{t}^{n}, x\right) \leq C_{T} .
$$

In particular, it follows that $\left(\mu_{t}^{n}\right)_{n}$ is tight for any $t \in[0, T]$. Moreover for $0 \leq s<t \leq T$, and any $\phi \in W^{1, \infty}$, $\|\phi\|_{W^{1, \infty}} \leq 1$, we have using (3.21) that

$$
\begin{aligned}
\left(\mu_{t}^{n}-\mu_{s}^{n}, \phi\right)= & \int_{s}^{t}\left(\mu_{\tau}^{n}, g\left(\tau, \mu_{\tau}^{n}\right) \phi^{\prime}\right) \mathrm{d} \tau-\int_{s}^{t}\left(\mu_{\tau}^{n}, d\left(\tau, \mu_{\tau}^{n}\right) \phi\right) \mathrm{d} \tau+\int_{s}^{t}\left(\mu_{\tau}^{n}, \beta\left(\tau, \mu_{\tau}^{n}\right)\right) \phi(0) \mathrm{d} \tau \\
& +\int_{s}^{t}\left(K\left[\mu_{\tau}^{n}\right], \phi\right)+\left(F\left[\mu_{\tau}^{n}\right], \phi\right) \mathrm{d} \tau \\
\leq & 3 \zeta C_{T}(t-s)+\int_{s}^{t} 3\left\|k^{n}\right\|_{\infty}\|\phi\|_{\infty}+C\|\phi\|_{\infty} \mathrm{d} \tau \leq \bar{C}_{T}(t-s) .
\end{aligned}
$$

Thus, $\left\|\mu_{t}^{n}-\mu_{s}^{n}\right\|_{\mathrm{BL}} \leq \bar{C}_{T}(t-s)$ so that the sequence $\left(\mu^{n}\right)_{n} \subset C\left([0, T], \mathcal{M}\left(\mathbb{R}^{+}\right)\right)$is uniformly equicontinuous. By the Arzela-Ascoli theorem, for any $T>0$, we therefore have a convergent subsequence (not relabeled) of the $\mu_{t}^{n}$ in $C\left([0, T], \mathcal{M}^{+}\left(\mathbb{R}^{+}\right)\right)$which converges to some $\mu \in C\left([0, T], \mathcal{M}^{+}\left(\mathbb{R}^{+}\right)\right)$. A diagonal argument gives that $\mu^{n} \rightarrow \mu$ in $C\left([0, T], \mathcal{M}^{+}\left(\mathbb{R}^{+}\right)\right)$for any $T>0$.

Since $\phi_{R}$ is bounded Lipschitz, we can pass to the limit $n \rightarrow \infty$ in $\left(\mu_{t}^{n}, \phi_{R}\right) \leq\left(\mu_{t}^{n}, x\right) \leq C_{T}$ to obtain $\left(\mu_{t}, \phi_{R}\right) \leq C_{T}$. Sending $R \rightarrow \infty$ gives that for any $T>0$,

$$
\left(\mu_{t}, x\right) \leq C_{T} \quad \text { for any } t \in[0, T] .
$$

We now want to pass to the limit $n \rightarrow \infty$ in the equation satisfied by $\mu^{n}$, namely

$$
\begin{aligned}
\int_{\mathbb{R}^{+}} & \phi(t, x) \mu_{t}^{n}(\mathrm{~d} x)-\int_{\mathbb{R}^{+}} \phi(0, x) \mu_{0}^{n}(\mathrm{~d} x) \\
= & \int_{0}^{t} \int_{\mathbb{R}^{+}}\left[\partial_{t} \phi(s, x)+g^{n}\left(s, \mu_{s}^{n}\right)(x) \partial_{x} \phi(s, x)-d^{n}\left(s, \mu_{s}^{n}\right)(x) \phi(s, x)\right] \mu_{s}^{n}(\mathrm{~d} x) \mathrm{d} s \\
& +\int_{0}^{t}\left(K^{n}\left[\mu_{s}^{n}\right]+F^{n}\left[\mu_{s}^{n}\right], \phi(s, \cdot)\right) \mathrm{d} s+\int_{0}^{t} \int_{\mathbb{R}^{+}} \phi(s, 0) \beta^{n}\left(s, \mu_{s}^{n}\right)(x) \mu_{s}^{n}(\mathrm{~d} x) \mathrm{d} s .
\end{aligned}
$$

Let $\phi \in C_{c}\left(\mathbb{R}^{+} \times \mathbb{R}^{+}\right)$. We pass to the limit in the right-hand side using that $\mu_{t}^{n} \rightarrow \mu_{t}$ for any $t \geq 0$. Since $\kappa^{n} \rightarrow \kappa$ uniformly on compact sets, $\left(\mu_{s}^{n}, 1\right) \leq C_{T}$, and $\mu_{s}^{n} \otimes \mu_{s}^{n} \rightarrow \mu_{s} \otimes \mu_{s}$ weakly, we can pass to the limit

$$
\begin{aligned}
2\left(K\left[\mu_{s}^{n}\right], \phi\right)= & \int_{\mathbb{R}^{+}} \int_{\mathbb{R}^{+}}\left(\kappa^{n}(x, y)-\kappa(x, y)\right)(\phi(x+y)-\phi(x)-\phi(y)) \mu_{s}^{n}(\mathrm{~d} x) \mu_{s}^{n}(\mathrm{~d} y) \\
& +\int_{\mathbb{R}^{+}} \int_{\mathbb{R}^{+}} \kappa(x, y)(\phi(x+y)-\phi(x)-\phi(y)) \mu_{s}^{n}(\mathrm{~d} x) \mu_{s}^{n}(\mathrm{~d} y) \\
\rightarrow & \int_{\mathbb{R}^{+}} \int_{\mathbb{R}^{+}} \kappa(x, y)(\phi(x+y)-\phi(x)-\phi(y)) \mu_{s}(\mathrm{~d} x) \mathrm{d} \mu_{s}(\mathrm{~d} y)=2\left(K\left[\mu_{s}\right], \phi\right) .
\end{aligned}
$$

Since $\left|\left(K\left[\mu_{s}^{n}\right], \phi\right)\right| \leq C$, we obtain by dominated convergence that

$$
\int_{0}^{t}\left(K\left[\mu_{s}^{n}\right], \phi\right) \mathrm{d} s \rightarrow \int_{0}^{t}\left(K\left[\mu_{s}\right], \phi\right) \mathrm{d} s .
$$


Similarly, we can pass to the limit in $\left(F\left[\mu_{s}^{n}\right], \phi\right)$ in the same way. Finally, in view of (S4), (3.21) and since $\phi$ has compact support we have for any $s \geq 0$ that

$$
\begin{aligned}
\int_{\mathbb{R}^{+}} d^{n}\left(s, \mu_{s}^{n}\right)(x) \phi(s, x) \mu_{s}^{n}(\mathrm{~d} x) & =\int_{\mathbb{R}^{+}}\left[d^{n}\left(s, \mu_{s}^{n}\right)(x)-d\left(s, \mu_{s}\right)(x)\right] \phi(s, x) \mu_{s}^{n}(\mathrm{~d} x)+\int_{\mathbb{R}^{+}} d\left(s, \mu_{s}\right)(x) \phi(s, x) \mu_{s}^{n}(\mathrm{~d} x) \\
& \rightarrow \int_{\mathbb{R}^{+}} d\left(s, \mu_{s}\right)(x) \phi(s, x) \mu_{s}(\mathrm{~d} x) .
\end{aligned}
$$

Since moreover

$$
\left|\int_{\mathbb{R}^{+}} d^{n}\left(s, \mu_{s}^{n}\right)(x) \phi(s, x) \mu_{s}^{n}(\mathrm{~d} x)\right| \leq \zeta\|\phi\|_{\infty}\left(\mu_{s}^{n}, 1\right) \leq C_{T}
$$

we obtain by the Dominated Convergence Theorem that

$$
\int_{0}^{t} \int_{\mathbb{R}^{+}} d^{n}\left(s, \mu_{s}^{n}\right)(x) \phi(s, x) \mu_{s}^{n}(\mathrm{~d} x) \mathrm{d} s \rightarrow \int_{0}^{t} \int_{\mathbb{R}^{+}} d\left(s, \mu_{s}\right)(x) \phi(s, x) \mu_{s}(\mathrm{~d} x) \mathrm{d} s .
$$

We treat the terms with $g^{n}$ and $\beta^{n}$ in the same way.

\section{Interplay BetWEen Growth, COAGUlation, AND FRAGMENTATiOn PROCESSES}

In the recent paper [35], it was shown that the steady state solution of a size-structured population model (i.e. model (3.8) with $K \equiv F \equiv 0$ ) with positive model ingredients is absolutely continuous with respect to the Lebesgue measure. This leads naturally to studying the effect the physical processes of coagulation and fragmentation would have on such regularity. To this end, we denote by $l_{0}(t)$ the solution to

$$
\left\{\begin{array}{l}
\frac{\mathrm{d}}{\mathrm{d} t} l_{0}(t)=g(t, \mu(t))\left(l_{0}(t)\right), \\
l_{0}(0)=0 .
\end{array}\right.
$$

Also, for simplicity of notation, we will denote by

$$
\tilde{g}(t, x):=g\left(t, \mu_{t}\right)(x), \quad \tilde{\beta}(s):=\int_{0}^{\infty} \beta\left(s, \mu_{s}\right)(y) \mu_{s}(\mathrm{~d} y), \quad T_{s, t}:=T_{s, t}^{\tilde{g}}
$$

where we recall $T_{s, t}^{g}$ from equation (2.1). We also recall from equation (3.11) that

$$
S(s)\left[\mu_{s}\right]=\tilde{\beta}(s) \delta_{x=0} .
$$

Before we present the main theorem of the section, we will establish the following useful lemma:

Lemma 4.1. Since $\tilde{g}>0$, the map $\Phi: s \mapsto T_{s, t}(0)$ is a bijection from $[0, t]$ to $\left[0, l_{0}(t)\right]$. Moreover

$$
\Phi^{\prime}(s)=-\tilde{g}(s, 0) \exp \left\{\int_{s}^{t} \partial_{x} \tilde{g}\left(\tau, T_{s, \tau}(0)\right) \mathrm{d} \tau\right\} \quad \forall s \in[0, t] .
$$

Moreover for any $0<s \leq t, T_{s, t}:\left[0, l_{0}(s)\right] \rightarrow\left[0, l_{0}(t)\right]$ is a bijection with

$$
\frac{\mathrm{d}}{\mathrm{d} x} T_{s, t}(x)=\exp \left\{\int_{s}^{t} \partial_{x} \tilde{g}\left(\tau, T_{s, \tau}(x)\right) \mathrm{d} \tau\right\} .
$$


Proof. The bijection property of $\Phi$ follows from the uniqueness of trajectories and the definition of $l_{0}(t)$. As for (4.1), taking the derivative with respect to $s$ in $\frac{\mathrm{d}}{\mathrm{d} t} T_{s, t}(0)=\tilde{g}\left(t, T_{s, t}(0)\right)$ yields

$$
\frac{\mathrm{d}}{\mathrm{d} t}\left(\frac{\mathrm{d}}{\mathrm{d} s} T_{s, t}(0)\right)=\partial_{x} \tilde{g}\left(t, T_{s, t}(0)\right) \frac{\mathrm{d}}{\mathrm{d} s} T_{s, t}(0)
$$

Since $\tilde{g}$ is $C^{1}$ in $x$,

$$
\frac{\mathrm{d}}{\mathrm{d} s} T_{s, t}(0)=\frac{\mathrm{d}}{\mathrm{d} s} T_{s, t}(0)_{\mid t=s} \exp \left\{\int_{s}^{t} \partial_{x} \tilde{g}\left(\tau, T_{s, \tau}(0)\right) \mathrm{d} \tau\right\} .
$$

Since $T_{s, t}(0)=\int_{s}^{t} \tilde{g}\left(\tau, T_{s, \tau}(0)\right) \mathrm{d} \tau$ we have $\frac{\mathrm{d}}{\mathrm{d} s} T_{s, t}(0)_{\mid t=s}=-\tilde{g}(s, 0)$ and so we deduce (4.1).

The proof of (4.2) is identical, but with taking the derivative with respect to $x$ in $\frac{\mathrm{d}}{\mathrm{d} t} T_{s, t}(x)=\tilde{g}\left(t, T_{s, t}(x)\right)$ and using that $\frac{\mathrm{d}}{\mathrm{d} x} T_{s, s}(x)=1$.

In particular for any bounded measurable function $\phi:[0, \infty) \rightarrow \mathbb{R}$,

$$
\begin{aligned}
& \int_{0}^{t}\left(T_{s, t} \sharp S(s)\left[\mu_{s}\right], \phi\right) \mathrm{d} s=\int_{0}^{t} \tilde{\beta}(s) \phi(\Phi(s)) \mathrm{d} s \\
& =\int_{0}^{l_{0}(t)} \phi(x) \frac{\tilde{\beta}\left(\Phi^{-1}(x)\right)}{\tilde{g}\left(\Phi^{-1}(x), 0\right)} \exp \left\{-\int_{\Phi^{-1}(x)}^{t} \partial_{x} \tilde{g}\left(\tau, T_{\Phi^{-1}(x), \tau}(0)\right) \mathrm{d} \tau\right\} \mathrm{d} x,
\end{aligned}
$$

so that $\int_{0}^{t} T_{s, t \sharp S(s)}\left[\mu_{s}\right] \mathrm{d} s=\int_{0}^{t} T_{s, t} \sharp \tilde{\beta}(s) \delta_{0} \mathrm{~d} s$ is the function

$$
x \rightarrow 1_{\left[0, l_{0}(t)\right]}(x) \frac{\tilde{\beta}\left(\Phi^{-1}(x)\right)}{\tilde{g}\left(\Phi^{-1}(x), 0\right)} \exp \left\{-\int_{\Phi^{-1}(x)}^{t} \partial_{x} \tilde{g}\left(\tau, T_{\Phi^{-1}(x), \tau}(0)\right) \mathrm{d} \tau\right\} .
$$

We now make use of the following definition:

Definition 4.2. The family $\{b(y, \cdot): y \geq 0\}$ is said to be uniformly equi-integrable if for any $\varepsilon>0$, there exists $\delta>0$ such that for any $V \subset \mathbb{R}^{+}$measurable with $|V|<\delta$, there holds $b(y, V)=\int_{V} b(y, x) \mathrm{d} x<\varepsilon$.

With this definition and the above lemma, we are now in position to establish the main result of this section. The proof that we propose is inspired by Lemma 3.5 of [56] and Lemma 2.6 of [42]. However the presence of the growth term adds new difficulties and novel techniques are adopted to handle these difficulties.

Theorem 4.3. Assume (A1)-(A3), (K), (F1), (F2), and (B2) hold with $g\left(t, \mu_{t}\right) \in C^{1}\left(\mathbb{R}^{+}\right)$taking strictly positive values, and let $\mu_{t}$ be the solution to (3.8) for some some initial condition $\mu_{0}$. Moreover, assume each measure $b(y, \cdot), y \geq 0$, is absolutely continuous w.r.t. Lebesgue measure with density $b(y, x)$, and that the family $\{b(y, \cdot): y \geq 0\}$ is uniformly equi-integrable. Then, for any $t>0, \mu_{t}$ is absolutely continuous on $\left[0, l_{0}(t)\right)$ with respect to the Lebesgue measure (i.e. $\mu_{t} \ll \mathrm{d} x$ ).

Proof. Recall that the solution $\mu$ was obtained as a fixed point of the map $\Gamma$ defined in (3.17) namely

$$
\mu_{t}=T_{t} \sharp \mu_{0}+\int_{0}^{t} T_{s, t} \sharp\left(F^{+}\left[\mu_{s}\right]+\tilde{\beta}(s) \delta_{0}\right) \mathrm{d} s+\int_{0}^{t} T_{s, t} \sharp\left(K^{+}\left[\mu_{s}\right]-\tilde{A}(s, \cdot) \mu_{s}\right) \mathrm{d} s
$$

where $T_{s, t}$ is the flow of the vector field $(t, x) \rightarrow \tilde{g}(t, x):=g\left(t, \mu_{t}\right)(x)$, and

$$
\tilde{A}(t, x)=d\left(t, \mu_{t}\right)(x)+a(x)+\int_{\mathbb{R}^{+}} \kappa(x, y) \mu_{t}(\mathrm{~d} y) \geq 0 .
$$


Notice due to the positivity of the model functions

$$
\mu_{t} \leq T_{t} \sharp \mu_{0}+\int_{0}^{t} T_{s, t} \sharp\left(F^{+}\left[\mu_{s}\right]+\tilde{\beta}(s) \delta_{0}\right) \mathrm{d} s+\int_{0}^{t} T_{s, t} \sharp K^{+}\left[\mu_{s}\right] \mathrm{d} s .
$$

Given some $\delta>0$ and $s \in[0, t]$, let $\mathcal{A}_{s}$ be the family of subsets of $\left[0, l_{0}(s)\right)$ of the form

$$
A=T_{s, s_{1}}^{-1}\left(\cdots\left(T_{s_{n-1}, s_{n}}^{-1}\left(T_{s_{n}, t}^{-1}(E)-x_{n}\right)-x_{n-1}\right) \cdots\right)-x_{1}
$$

where $n \in \mathbb{N}_{0}, s \leq s_{1} \leq \ldots \leq s_{n} \leq t, x_{1}, \ldots, x_{n} \geq 0$, and $E \subset\left[0, l_{0}(t)\right)$ is a Borel subset with $|E|<\delta$. It is implicitly understood that at each step of the construction of $A$ we take the intersection with $[0, \infty)$. Define then

$$
\mathcal{E}(s):=\sup \left\{\mu_{s}(A): A \in \mathcal{A}_{s}\right\}
$$

where we extend $\mu_{s}$ to $(-\infty, 0)$ by 0 .

Notice that $T_{s} \sharp \mu_{0}$ is supported in $\left[l_{0}(s), \infty\right)$ and that any $A \in \mathcal{A}_{s}$ is a subset of $\left[0, l_{0}(s)\right)$. It follows that for any $A \in \mathcal{A}_{s}$ of the form (4.6) we have by (4.5) that

$$
\mu_{s}(A) \leq \int_{0}^{s}\left(F^{+}\left[\mu_{\tau}\right]+\tilde{\beta}(\tau) \delta_{0}\right)\left(T_{\tau, s}^{-1}(A)\right) \mathrm{d} \tau+\int_{0}^{s} K^{+}\left[\mu_{\tau}\right]\left(T_{\tau, s}^{-1}(A)\right) \mathrm{d} \tau .
$$

For any $0 \leq a \leq b \leq T$ and any subset $B \subset[0, \infty)$ we have by (4.2) and assumption (A2) that

$$
\left|T_{a, b}^{-1}(B)\right|=\int_{\mathbb{R}^{+}} 1_{B}\left(T_{a, b}(y)\right) \mathrm{d} y=\int_{\mathbb{R}^{+}} 1_{B}(x)\left|\frac{\mathrm{d}}{\mathrm{d} x} T_{a, b}^{-1}(x)\right| \mathrm{d} x \leq e^{\zeta(b-a)}|B| .
$$

Using the translation invariance of Lebesgue measure we then have that the measure of $A$ given by (4.6) can be bounded by

$$
|A| \leq e^{\zeta\left(\left(t-s_{n}\right)+\left(s_{n}-s_{n-1}\right)+\ldots+\left(s_{1}-s\right)\right)}|E| \leq e^{\zeta(t-s)} \delta \leq C_{T} \delta
$$

Here and in the sequel of the proof, we denote by $C_{T}$ any constant depending only on $T$ and the constants appearing in assumptions (A1), (A2), (A3), (K), (F1), and (F2). It then follows from (4.4), (A2), (A3) that

$$
\int_{0}^{s} \tilde{\beta}(\tau) \delta_{0}\left(T_{\tau, s}^{-1}(A)\right) \mathrm{d} \tau \leq C_{T} \delta .
$$

Moreover

$$
F^{+}\left[\mu_{\tau}\right](A)=\int_{\mathbb{R}^{+}} b(y)(A) a(y) \mu_{\tau}(\mathrm{d} y) \leq\|a\|_{\infty}\left\|\mu_{\tau}\right\|_{\mathrm{TV}} \sup _{y \geq 0} b(y)(A) .
$$

Since $\left\|\mu_{\tau}\right\|_{\mathrm{TV}} \leq C_{T}, \tau \in[0, s]$, we obtain

$$
\int_{0}^{s} F^{+}\left[\mu_{\tau}\right]\left(T_{\tau, s}^{-1}(A)\right) \mathrm{d} \tau \leq C_{T} \sup _{y \geq 0,|A| \leq C_{T} \delta} b(y)(A) .
$$

If we assume that the family $\{b(y, \cdot)\}_{y \geq 0}$ is uniformly equi-integrable then $\sup _{y \geq 0,|A| \leq C_{T} \delta} b(y)(A)$ goes to 0 as $\delta \rightarrow 0$. We denote $o(1)$ any quantity going to 0 as $\delta \rightarrow 0$ uniformly in $t \in[0, T]$ and $A$. Coming back to (4.7) we thus obtained so far that

$$
\mu_{s}(A) \leq o(1)+\int_{0}^{s} K^{+}\left[\mu_{\tau}\right]\left(T_{\tau, s}^{-1}(A)\right) \mathrm{d} \tau
$$

To bound the coagulation term in the right-hand side recall the definition of $K^{+}$:

$$
2 K^{+}\left[\mu_{\tau}\right]\left(T_{\tau, s}^{-1}(A)\right)=\int_{\mathbb{R}^{+}} 1_{T_{\tau, s}^{-1}(A)}(z+y) \kappa(x, y) \mu_{\tau}(\mathrm{d} z) \mu_{\tau}(\mathrm{d} y) \leq\|\kappa\|_{\infty} \int_{\mathbb{R}^{+}} \mu_{\tau}\left(T_{\tau, s}^{-1}(A)-y\right) \mu_{\tau}(\mathrm{d} y) .
$$


Since $T_{\tau, s}^{-1}(A)-y \in \mathcal{A}_{\tau}$ we obtain

$$
2 K^{+}\left[\mu_{\tau}\right]\left(T_{s, t}^{-1}(A)\right) \leq\|\kappa\|_{\infty} \mathcal{E}(\tau) \int_{\mathbb{R}^{+}} \mu_{\tau}(\mathrm{d} y)
$$

so that

$$
K^{+}\left[\mu_{\tau}\right]\left(T_{\tau, s}^{-1}(A)\right) \leq C_{T} \mathcal{E}(\tau)
$$

Coming back to (4.8) we obtain

$$
\mu_{s}(A) \leq o(1)+C_{T} \int_{0}^{s} \mathcal{E}(\tau) \mathrm{d} \tau .
$$

Since this holds for any $A \in \mathcal{A}_{s}$ and any $s \leq t$ we deduce

$$
\mathcal{E}(t) \leq o(1)+C_{T} \int_{0}^{t} \mathcal{E}(\tau) \mathrm{d} \tau
$$

which yields by Gronwall inequality

$$
\mathcal{E}(t)=o(1)
$$

In particular, since $E \in \mathcal{A}_{t}$,

$$
\mu_{t}(E)=o(1) \quad \forall E \subset\left[0, l_{0}(t)\right),|E|<\delta .
$$

It follows that $\mu_{t}$ is absolutely continuous on $\left[0, l_{0}(t)\right)$ for any $t>0$.

This leads us to the following corollary about the regularity of a steady state solution to model (3.8).

Corollary 4.4. Let the assumptions of Theorem 4.3 hold with $g, d, \beta$ dependent on time only through $\mu_{t}$ (i.e. $g\left(t, \mu_{t}\right)=g\left(\mu_{t}\right)$ etc.) and assume $\mu \in \mathcal{M}^{+}\left(\mathbb{R}^{+}\right)$is a steady state solution of model (3.8). Then $\mu$ is absolutely continuous with respect to the Lebesgue measure. Furthermore, $\mu$ satisfies

$$
\int_{\mathbb{R}^{+}} g(\mu)(x) \mu(\mathrm{d} x)=\int_{\mathbb{R}^{+}} x d(\mu)(x) \mu(\mathrm{d} x) .
$$

Proof. The proof follows from similar arguments of Proposition 2.6 in [35] with making use of $g(\mu)(x)>0$ for all $x$. Indeed, since $g(\mu)(x)>0$ for all $x$ we have

$$
\lim _{t \rightarrow \infty} l^{0}(t)=\infty
$$

Theorem 4.3 then implies a solution $\mu_{t}$ is absolutely continuous on the interval $\left[0, l^{0}(t)\right)$. Thus, the steady state solution $\mu_{t}=\mu$ is absolutely continuous on $[0, \infty)$. The mass conservation equation follows from Theorem 3.4.

\section{From measure equation to Discrete And CONTINuOUs Equations}

It is often claimed that one of the many benefits of population models set in measure spaces is the unification of the study of discrete and continuous structure. In this section, we demonstrate this property by showing that model (3.8) includes as special cases the discrete Smoluchowski equations [55] and the continuous Müller model [49]. 


\subsection{Continuous density model}

In this subsection, we briefly demonstrate how model (3.8) reduces to model (1.2) and hence encompasses continuous density models studied in $[1,3,15,49]$ from $(3.8)$. This follows naturally under the following assumptions:

(B1) $\mu_{0}$ is absolutely continuous with respect to the Lebesgue measure,

(B2) $b(y, \cdot)$ is absolutely continuous with respect to the Lebesgue measure.

Then by undoing the derivations of (3.1) and (3.6), one arrives at the density equations (3.4) and (3.7) covered in the aforementioned works. In particular, we can recover the binary fragmentation kernels studied in $[1,15,37]$ by taking

$$
a(y)=\frac{1}{2} \int_{0}^{y} \gamma(y-s, s) \mathrm{d} s, \quad b(y, \cdot)=\frac{\gamma(x, y-x)}{a(y)} \mathrm{d} x
$$

where the function $\gamma(x, y)$ models the rate at which a particles of size $x+y$ fragment into particles of size $x$ and $y$.

\subsection{Discrete equation}

In this subsection, we show under certain assumptions, model (3.8) will reduce to the discrete coagulationfragmentation equation (1.1) and hence covers models discussed in $[10,55]$. To obtain these equations, we set $g(t, \mu)=\beta(t, \mu) \equiv 0$ for the remainder of this section. To this end, suppose that the measures $\mu_{0}$ and $b(y, \cdot)$ are supported on $h \mathbb{N}=\{h, 2 h, \ldots\}$ for some fixed $h>0$ i.e.

(C1) $\mu_{0}=\sum_{i \in \mathbb{N}} m_{i}^{0} \delta_{i h}$ where for each $i, m_{i}^{0} \in \mathbb{R}^{+}$,

(C2) $b(y, \cdot)=\sum_{i \in \mathbb{N}} b_{i}(y) \delta_{i h}$.

We then have the following result:

Theorem 5.1. Let assumptions (A1), (A2), (K), (F1), (F2), (C1), (C2), and (C3) hold. Then for any $t \in$ $[0, \infty)$, the solution $\mu_{t}$ of $(3.8)$ is supported on $h \mathbb{N}_{0}$ :

$$
\mu_{t}=\sum_{l \in \mathbb{N}} m_{l}(t) \delta_{l h},
$$

where the $m_{l}(t), l \in \mathbb{N}$, satisfy the discrete coagulation-fragmentation equation

$$
\begin{aligned}
\frac{\mathrm{d}}{\mathrm{d} t} m_{l}(t)+d\left(t, \mu_{t}\right)(l h) m_{l}(t)= & \frac{1}{2} \sum_{i=1}^{l-1} m_{i}(t) m_{l-i}(t) \kappa(i h,(l-i) h)-\sum_{i=1}^{\infty} \kappa(i h, l h) m_{i}(t) m_{l}(t) \\
& +\sum_{i \geq l} b_{l}(i h) a(i h) m_{i}(t)-a(l h) m_{l}(t)
\end{aligned}
$$

with initial condition $m_{l}(0)=m_{l}^{0}$.

Proof. It is clear from Theorem 3.4 that (3.8) has a unique solution $\mu \in C\left([0, \infty), \mathcal{M}^{+}\left(\mathbb{R}^{+}\right)\right)$. Moreover, according to the proof of Theorem 3.4, $\mu$ is a fixed-point of $\Gamma$ defined in (3.17). Since $g=0, T_{s, t}^{g}$ is the identity map. Thus $\Gamma$ is simply given by

$$
\Gamma[\nu]_{t}=\mu_{0}+\int_{0}^{t}\left\{F\left[\nu_{s}\right]+K\left[\nu_{s}\right]+S(s)\left[\nu_{s}\right]-d\left(s, \nu_{s}\right) \nu_{s}\right\} \mathrm{d} s
$$

for any $\nu \in C\left([0, \infty), \mathcal{M}\left(\mathbb{R}^{+}\right)\right)$. Notice that if $\nu_{t}$ is supported in $h \mathbb{N}$ for any $s$ then so is $\Gamma[\nu]_{t}$ (concerning $K^{+}$ notice this follows from the fact that $h \mathbb{N}+h \mathbb{N} \subset h \mathbb{N}$ ). We can thus replace $X_{T}$ in (3.16) by

$$
X_{T}=\left\{\mu \in C([0, T], \mathcal{M}(h \mathbb{N})): \mu(0)=\mu_{0},\|\mu\|_{\mathrm{TV}} \leq 2\left\|\mu_{0}\right\|_{\mathrm{TV}} \forall t \in[0, T]\right\},
$$


and repeat the proof of Theorem 3.4 verbatim to obtain that $\mu_{t}$ is supported in $h \mathbb{N}$ for any $t \geq 0$. It follows that $\mu_{t}$ can be written as in (5.2). Equation (5.3) follows from (3.10) taking a $C^{1}$ test-function, $\phi$, constant in time and supported in $(l h-h, l h+h)$ such that $\phi(l h)=1$.

\section{Numerical methods AND RESults}

In this section, we present a semidiscrete scheme for a coagulation-fragmentation equation based on (5.3) and Theorem 5.1 as well as provide some numerical results based on this scheme. For the rest of this section, we assume that $\beta(t, \mu)=g(t, \mu) \equiv 0$.

\subsection{A semi-discrete numerical scheme}

We consider equation (3.8) with $\int_{\mathbb{R}^{+}}(1+x) \mu_{0}(\mathrm{~d} x)<\infty$ and we assume that assumptions (A1), (A2), (A3), $(\mathrm{K}),(\mathrm{F} 1)$, and (F2) hold. We present a semi-discrete scheme inspired by [44].

Consider the grid $h \mathbb{N}_{0}$ and the cell $\Lambda^{h}(i)$ centered at the grid point $i h$ defined by

$$
\Lambda^{h}(i):=[h i-h / 2, h i+h / 2), i \geq 1, \quad \Lambda^{h}(0)=[0, h / 2) .
$$

We define the discretization of the initial condition $\mu_{0} \in \mathcal{M}^{+}\left(\mathbb{R}^{+}\right)$with respect to the grid $h \mathbb{N}_{0}$ by

$$
\mu_{0}^{h}=\sum_{i \geq 0} \mu_{0}^{h}(i) \delta_{h i}, \quad \mu_{0}^{h}(i)=\mu_{0}\left(\Lambda^{h}(i)\right) .
$$

We want to approximate the solution $\mu_{t}$ of (3.8) by measures $\mu_{t}^{h}$ supported in $h \mathbb{N}_{0}$ and solution of some discretized equation. We first approximate the model coefficients $\kappa, a, b$ as follow. First we define

$$
a_{i}^{h}=\frac{1}{h} \int_{\Lambda^{h}(i)} a(y) \mathrm{d} y, \quad \kappa_{i, j}^{h}=\frac{1}{h^{2}} \int_{\Lambda^{h}(i) \times \Lambda^{h}(j)} \kappa(x, y) \mathrm{d} x \mathrm{~d} y
$$

for $i, j \geq 1$, and

$$
a_{0}^{h}=\frac{2}{h} \int_{\Lambda^{h}(0)} a(y) \mathrm{d} y, \quad \kappa_{0,0}^{h}=\frac{4}{h^{2}} \int_{\Lambda^{h}(0) \times \Lambda^{h}(0)} \kappa(x, y) \mathrm{d} x \mathrm{~d} y
$$

(with the natural modifications for $\kappa_{0, j}^{h}$ and $\kappa_{i, 0}^{h}, i \geq 1$ ). We then let $a^{h} \in W^{1, \infty}\left(\mathbb{R}^{+}\right)$and $\kappa^{h} \in W^{1, \infty}\left(\mathbb{R}^{+} \times \mathbb{R}^{+}\right.$) be the linear interpolation of the $a_{i}^{h}$ and $\kappa_{i, j}^{h}$ respectively. Finally, we define the measure $b^{h}(j h, \cdot) \in \mathcal{M}^{+}(h \mathbb{N})$ by

$$
b^{h}(j h, \cdot)=\sum_{i \leq j} b\left(j h, \Lambda^{h}(i)\right) \delta_{i h}
$$

and then $b^{h}(x, \cdot) \in \mathcal{M}^{+}\left(h \mathbb{N}_{0}\right)$ for $x \geq 0$ as the linear interpolate between the $b^{h}(j h, \cdot)$. We define the corresponding coagulation and fragmentation operators $K^{h}$ and $F^{h}$ by

$$
\begin{aligned}
\left(K^{h}[\mu], \phi\right) & =\frac{1}{2} \int_{\mathbb{R}^{+}} \int_{\mathbb{R}^{+}} \kappa^{h}(y, x)[\phi(x+y)-\phi(x)-\phi(y)] \mu(\mathrm{d} x) \mu(\mathrm{d} y), \\
F^{h}[\mu](\cdot) & =\int_{\mathbb{R}^{+}} b^{h}(y, \cdot) a^{h}(y) \mu(\mathrm{d} y)-a^{h} \mu .
\end{aligned}
$$

Notice that $K^{h}, a^{h}, b^{h}$ satisfy (K), (F1), (F2)(i), (F2)(ii), (C1), (C2), (C3). However (F2)(iii) is only satisfied up to an error of order $h$, namely

$$
\left|\left(b^{h}(y, \cdot), x\right)-y\right| \leq C h \quad \text { for any } y \geq 0,
$$


where the constant $C$ depends only on $C_{b}$ given by (F2)(i). Indeed recalling that for any $j \geq 0$ the measure $b(j h, \cdot)$ is non-negative and supported in $[0, j h]$ we have

$$
\begin{aligned}
\left|\left(b^{h}(j h, \cdot), x\right)-j h\right| & =\left|\left(b^{h}(j h, \cdot), x\right)-(b(j h, \cdot), x)\right| \leq \sum_{i \leq j} \int_{\Lambda^{h}(i)}|i h-x| b(j h, \mathrm{~d} x) \\
& \leq \frac{h}{2} b\left(j h, \mathbb{R}^{+}\right) \leq \frac{1}{2} C_{b} h .
\end{aligned}
$$

The result follows recalling that for $y \in[j h,(j+1) h]$ we have $b^{h}(y, \cdot)=\frac{1}{h}\left[b^{h}((j+1) h, \cdot)-b^{h}(j h, \cdot)\right](y-j h)+$ $b^{h}(j h, \cdot)$.

It then follow from Theorem 5.1 that (3.8) with $g=d=\beta=0, K=K^{h}, F=F^{h}$ has a unique solution $\mu \in C\left([0, \infty), \mathcal{M}^{+}\left(\mathbb{R}^{+}\right)\right)$which is supported on $h \mathbb{N}$ :

$$
\mu_{t}^{h}=\sum_{l \in \mathbb{N}_{0}} m_{l}^{h}(t) \delta_{l h},
$$

where the $m_{l}^{h}(t), l \in \mathbb{N}_{0}$, satisfy the discrete coagulation-fragmentation equation

$$
\begin{aligned}
\frac{\mathrm{d}}{\mathrm{d} t} m_{l}^{h}(t)= & \frac{1}{2} \sum_{i=1}^{l-1} m_{i}^{h}(t) m_{l-i}^{h}(t) \kappa_{i, l-i}^{h}-\sum_{i=1}^{\infty} \kappa_{i, l}^{h} m_{i}^{h}(t) m_{l}^{h}(t) \\
& +\sum_{i \geq l} b\left(i h, \Lambda^{h}(l)\right) a_{i}^{h} m_{i}^{h}(t)-a_{l}^{h} m_{l}^{h}(t)
\end{aligned}
$$

with initial condition $m_{l}^{h}(0)=m_{0}^{h}(l)$. Notice that the first two terms on the right hand side of (6.2) make up the discrete Smoluchowski equations and therefore these terms conserve mass. Indeed, multiplying by $x_{l}:=l h$ and summing over $l=1,2, \ldots$ we have

$$
\begin{aligned}
& \frac{1}{2} \sum_{l=1}^{\infty} \sum_{i=1}^{l-1} x_{l} m_{i}^{h}(t) m_{l-i}^{h}(t) \kappa_{i, l-i}^{h}-\sum_{l=1}^{\infty} \sum_{i=1}^{\infty} x_{l} \kappa_{i, l}^{h} m_{i}^{h}(t) m_{l}^{h}(t) \\
& \quad=\frac{1}{2} \sum_{i=1}^{\infty} \sum_{j=1}^{\infty}\left(x_{i}+x_{j}\right) m_{i}^{h}(t) m_{j}^{h}(t) \kappa_{i, j}^{h}-\sum_{l=1}^{\infty} \sum_{i=1}^{\infty} x_{l} \kappa_{i, l}^{h} m_{i}^{h}(t) m_{l}^{h}(t) \\
& \quad=0 .
\end{aligned}
$$

However, since $\left|\left(b^{h}(y, \cdot), x\right)-y\right|=O(h)$ it is clear that the fragmentation terms only conserve mass up to an error of order $h$.

To study the limit of $\mu_{t}^{h}$ as $h \rightarrow 0$ we first state the following properties:

Proposition 6.1. The following holds:

(i) $\lim _{h \rightarrow 0}\left\|\mu_{0}^{h}-\mu_{0}\right\|_{\mathrm{BL}}=0$ and $\int_{\mathbb{R}^{+}}(1+x) \mu_{0}^{h}(\mathrm{~d} x) \leq C$,

(ii) $a^{h} \rightarrow a, \kappa^{h} \rightarrow \kappa$ uniformly on compact sets, and $a^{h}, \kappa^{h} \leq C$,

(iii) for any $\phi \in W^{1, \infty}(\mathbb{R}),\left(b^{h}(x), \phi\right) \rightarrow(b(x), \phi)$ uniformly for $x$ in a compact set.

Proof. For any $\phi \in W^{1, \infty}\left(\mathbb{R}^{+}\right),\|\phi\|_{W^{1, \infty}} \leq 1$, we have

$$
\left(\mu_{0}^{h}-\mu_{0}, \phi\right)=\sum_{i \geq 0} \int_{\Lambda_{i}(h)} \phi(i h)-\phi(x) \mu_{0}(\mathrm{~d} x) \leq \sum_{i \geq 0} \int_{\Lambda_{i}(h)}|i h-x| \mu_{0}(\mathrm{~d} x) \leq \frac{h}{2} \mu_{0}\left(\mathbb{R}^{+}\right) .
$$

Moreover

$$
\int_{\mathbb{R}^{+}} x \mu_{0}^{h}(\mathrm{~d} x)=\sum_{i \geq 0} \int_{\Lambda^{h}(i)} i h \mu_{0}(\mathrm{~d} x)=\sum_{i \geq 0} \int_{\Lambda^{h}(i)} x \mu_{0}(\mathrm{~d} x)+O(h)=\left(\mu_{0}, x\right)+O(h)
$$


which proves (i).

Concerning (ii), since $0 \leq a, \kappa \leq C$, we have $0 \leq a^{h}, \kappa^{h} \leq C$. Moreover, let $x \in[n h, m h]$ for some $n \neq m \in \mathbb{N}_{0}$. Then letting $\chi_{A}(x)$ represent the characteristic function over the set $A$, we have

$$
\begin{aligned}
\left\|a^{h}-a\right\|_{\infty} & \leq \sum_{i=n}^{m}\left|\left(a_{i+1}^{h}-a_{i}^{h}\right)\left(\frac{x-i h}{h}\right)+a_{i}-a(x)\right| \chi_{[i h,(i+1) h)}(x) \\
& \leq \sum_{i=n}^{m}\left|a\left(x_{i+1}\right)-a\left(x_{i}\right)+a\left(x_{i}\right)-a(x)+O(h)\right| \chi_{[i h,(i+1) h)}(x) \\
& \leq\|a\|_{W^{1, \infty}} 2 h(m-n)+O(h) .
\end{aligned}
$$

Finally for (iii) again assume $x \in[n h, m h]$, then for $\phi \in W^{1, \infty}(\mathbb{R})$ we have

$$
\begin{aligned}
\left(b^{h}(x)-b(x), \phi\right) & =\sum_{j=n}^{m}\left[\left(b_{j+1}^{h}-b_{j}^{h}, \phi\right)\left(\frac{x-j h}{h}\right)+\left(b_{j}^{h}-b(x), \phi\right)\right] \chi_{[i h,(i+1) h)}(x) \\
& \leq \sum_{j=n}^{m}\left[\sum_{i \leq j+1} b\left((j+1) h, \Lambda^{h}(i)\right) \phi(i h)-\sum_{i \leq j} b\left((j) h, \Lambda^{h}(i)\right) \phi(i h)+\left(b_{j}^{h}-b(x), \phi\right)\right] \\
& =\sum_{j=n}^{m}[(b((j+1) h)-b(j h), \phi)+(b(j h)-b(x), \phi)+O(h)] \chi_{[i h,(i+1) h)}(x) .
\end{aligned}
$$

Making use of assumption (F2), we have

$$
\left(b^{h}(x)-b(x), \phi\right) \leq 2 L_{b}\|\phi\|_{W^{1, \infty}} h|m-n|,
$$

which completes the proof.

It follows form this proposition that the assumption of Theorem 3.6 are satisfied. Thus, we deduce that $\mu^{h}$ converges along a subsequence $h \rightarrow 0$ to $\mu$ solution of equation (3.8). Since this equation has a unique solution, the whole sequence $\mu^{h}$ converges to $\mu$ :

Theorem 6.2. The measure $\mu_{t}^{h}=\sum_{i \geq 0} m_{i}^{h}(t) \delta_{i h}$ where the $m_{i}^{h}$ solve (6.2) converges to the solution $\mu_{t}$ of equation (3.8) in $C\left([0, T], \mathcal{M}\left(\mathbb{R}^{+}\right)\right)$for any $T>0$.

We can thus think of the system (6.2) as a semi-discrete scheme for solving equation (3.8). One could combine this semidiscrete scheme with any ordinary differential equation scheme (e.g. any Runge-Kutta Method) to arrive at a fully discrete scheme. Convergence for such a scheme then follows from a standard triangle inequality argument. In the next section we present some numerical experiments to evaluate the quality of such a scheme.

Remark 6.3. One can easily include the case $\beta, d>0$ as these terms do not affect the discrete structure of the solution. However, in the case of additionally assuming $g>0$, it is not true that the solution is discrete for all time. This result was shown for structured population models (without coagulation and fragmentation) in [35] and with coagulation-fragmentation in Section 4.

\subsection{Mass conserving fragmentation term}

To remedy the error generated in mass conservation of the scheme discussed in the previous section, we propose a new approximation of $b(y, \mathrm{~d} x)$ in the form $b^{h}(y, \cdot)=\sum_{j=1}^{\infty} \alpha_{j}(y) \delta_{x_{j}}$ for which the following holds:

$$
\sum_{j=1}^{\infty} \alpha_{j}(y) x_{j}=(b(y, \cdot), x) .
$$


A natural choice of $\alpha_{j}(y)$ is given by

$$
\alpha_{j}(y)=\frac{1}{x_{j}} \int_{\Lambda^{h}(j)} x b(y, \mathrm{~d} x) .
$$

This approximation results in a mass conserving scheme at the expense of requiring a minimum positive size $x_{0}$. We have the following result:

Proposition 6.4. Assume there is a positive minimum size $x_{0}>0$ and therefore the points $x_{j}=x_{0}+j h$. Then

$$
\left\|b^{h}(y, \cdot)-b(y, \cdot)\right\|_{\mathrm{BL}} \longrightarrow 0 \quad \text { as } h \longrightarrow 0 .
$$

Proof. Taking $\phi(x) \in W^{1, \infty}(\mathbb{R})$ with $\|\phi\|_{W^{1, \infty}} \leq 1$ and letting $\phi_{j}:=\phi\left(x_{j}\right)$ we have

$$
\begin{aligned}
\left(b^{h}(y, \cdot)-b(y, \cdot), \phi\right) & =\sum_{i=1}^{\infty} \int_{\Lambda(i h)} \frac{\phi_{i}}{x_{i}} x-\phi(x) b(y, \mathrm{~d} x) \\
& =\sum_{i=1}^{\infty} \int_{\Lambda(i h)} \frac{\phi_{i} x-\phi(x) x_{i}}{x_{i}} b(y, \mathrm{~d} x) \\
& =\sum_{i=1}^{\infty} \int_{\Lambda(i h)} \frac{\phi_{i}\left(x-x_{i}\right)}{x_{i}}+\frac{\left(\phi_{i}-\phi(x)\right) x_{i}}{x_{i}} b(y, \mathrm{~d} x) .
\end{aligned}
$$

Since $0<x_{0} \leq x_{i}$ the first term is bounded and making use of the Lipschitz property of $\phi$ we have

$$
\begin{aligned}
\sum_{i=1}^{\infty} \int_{\Lambda(i h)} \frac{\phi_{i}\left(x-x_{i}\right)}{x_{i}}+\frac{\left(\phi_{i}-\phi(x)\right) x_{i}}{x_{i}} b(y, \mathrm{~d} x) & \leq \sum_{i=1}^{\infty} \int_{\Lambda(i h)}\left(\frac{\phi_{i}}{x_{0}}+1\right) \frac{h}{2} b(y, \mathrm{~d} x) \\
& \leq\left(\frac{1}{2 x_{0}}+\frac{1}{2}\right) C_{b} h .
\end{aligned}
$$

Therefore by the same arguments in the section above, we can conclude that a scheme with this term will converge to the solution of equation (3.8) with $g=d=\beta=0$.

The standard kernel taken for a structure domain $\mathbb{R}^{+}$is given by $b(y, \mathrm{~d} x)=\frac{2}{y} \mathrm{~d} x$. For the domain $\left[x_{0}, \infty\right)$, an example of a kernel which satisfies assumption (F2) is given by

$$
b(y, \mathrm{~d} x):=\frac{2 q}{y-x_{0}}\left(\frac{x-x_{0}}{y-x_{0}}\right)^{q-1} \mathrm{~d} x, \quad q=1-\frac{2 x_{0}}{y} .
$$

Notice, that if $x_{0}=0$, then the above kernel reduces to $\frac{2}{y} \mathrm{~d} x$. It should be noted that it is important to calculate $\alpha_{j}(y)$ exactly when implementing the scheme. Otherwise, numerical integration error may be introduced resulting in lack of mass conservation.

\subsection{Numerical results}

In this section, we test the semidiscrete scheme against some commonly used examples. We begin by testing the coagulation and fragmentation portions of the scheme separately. We implement the semidiscrete scheme using MATLAB's ode45 function. In each example, we present the exact solution at time $T=1$ plotted against the structure variable, $x$, the absolute value difference of the numerical and exact solution, and the relative mass between the numeric and exact solutions plotted against time. We remark that for examples with only coagulation, the semi-discrete scheme (6.2) conserves mass (i.e. (6.3)); therefore, any change of mass is due to simulating infinite domain problems over a finite interval. Where it is applicable, we provide a table calculating the BL-norm and numerical order of the scheme. The BL-norm is approximated by the algorithm provided in [34], while the numerical order of the scheme is calculated using the standard calculation:

$$
\log _{2}\left(\left\|\mu_{t}-\mu_{t}^{2 h}\right\|_{\mathrm{BL}} /\left\|\mu_{t}-\mu_{t}^{h}\right\|_{\mathrm{BL}}\right) \text {. }
$$



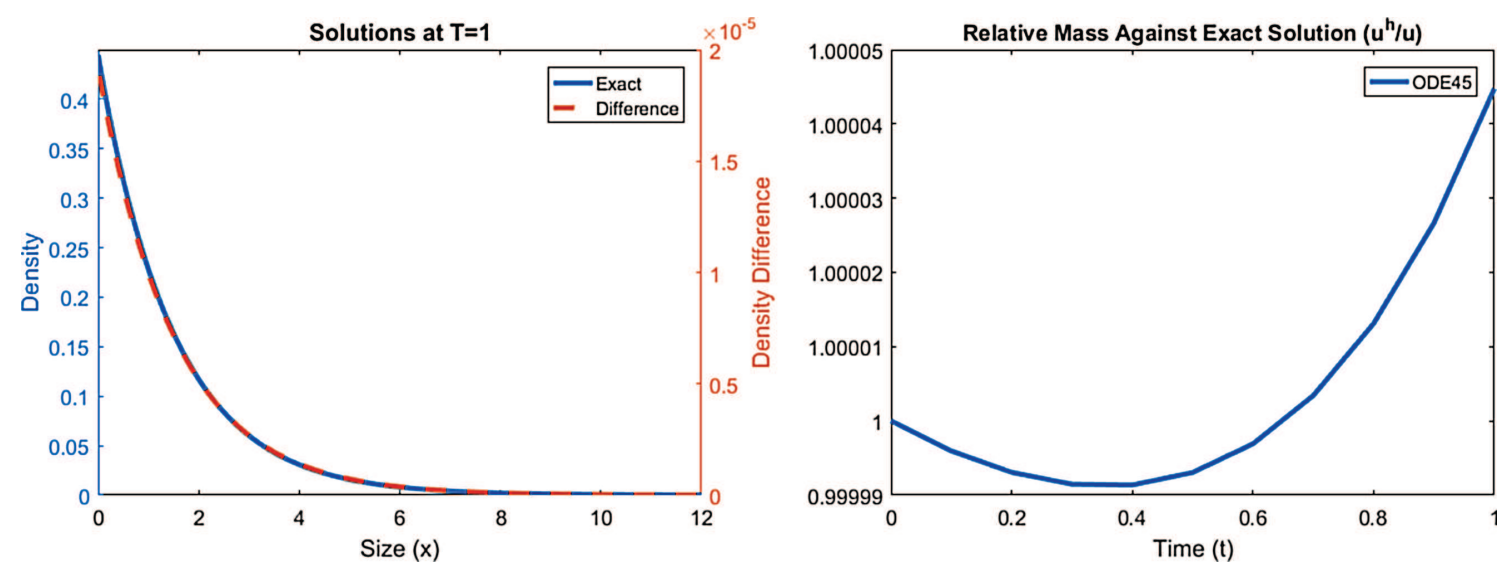

FiguRE 1. For Example 6.5 we present on the left side the exact solution (solid line) and the absolute value of the difference between the exact and numerical solution (dashed-line). On the right side we present the relative mass.

TABLE 1. Error and numerical order of convergence calculated for Example 6.5.

\begin{tabular}{lll}
\hline \hline Number of points & BL-error & Order \\
\hline 40 & 0.0072641 & NA \\
80 & 0.0019723 & 1.8809 \\
160 & 0.0005119 & 1.9459 \\
320 & 0.00013018 & 1.9754 \\
640 & 0.000032716 & 1.9924 \\
1280 & 0.0000080986 & 2.0143 \\
\hline
\end{tabular}

\subsubsection{Coagulation and fragmentation examples}

In this section we presented several numerical example focused on coagulation and fragmentation processes.

Example 6.5. For the first example, we take the coagulation kernel $\kappa(x, y) \equiv 1$ with $\mu_{0}=e^{-x} \mathrm{~d} x$ and all other model ingredients are set to 0 . This problem has an exact solution

$$
\mu_{t}=\left(\frac{2}{2+t}\right)^{2} \exp \left(-\frac{2}{2+t} x\right) \mathrm{d} x
$$

see [39] for more details. Numerical simulations for this example are presented in Figure 1 with $\Delta x=1 / 40$ and the BL error and order of conference are presented in Table 1. Simulation are performed over the finite domain $x \in[0,20]$.

Example 6.6. Although our theory does not cover the phenomenon of gelation, we include a numerical example showing how the semi discrete scheme handles such kernels. In this example, we take $\kappa(x, y)=x y$ with $\mu_{0}=$ $e^{-x} / x \mathrm{~d} x$. This has exact solution (see e.g. [39]).

$$
\mu_{t}=e^{-T x} \frac{I_{1}\left(2 x t^{1 / 2}\right)}{x^{2} t^{1 / 2}} \mathrm{~d} x,
$$

where

$$
T= \begin{cases}1+t & t \leq 1 \\ 2 t^{1 / 2} & \text { otherwise }\end{cases}
$$



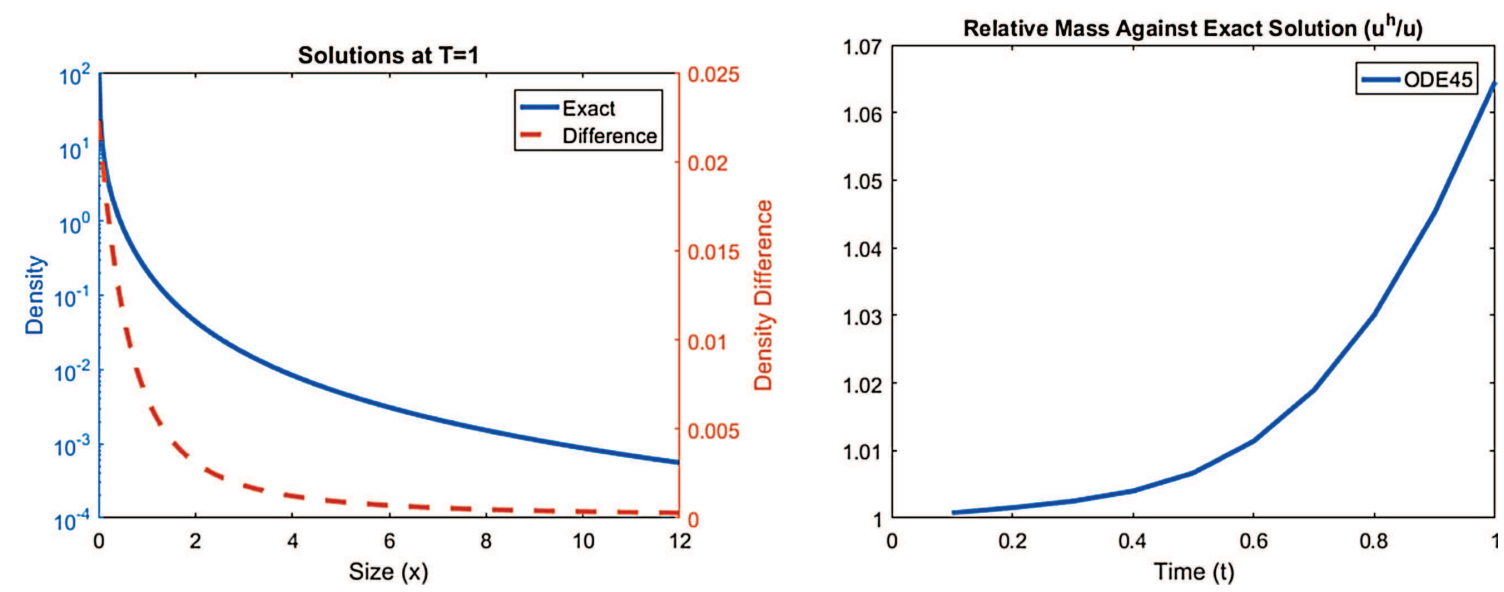

Figure 2. For Example 6.6 we present on the left side the exact solution (solid line) and the absolute value of the difference between the exact and numerical solution (dashed-line). On the right side we present the relative mass.

TABLE 2. Error and numerical order of convergence Example 6.7.

\begin{tabular}{lllll}
\hline \hline \multirow{2}{*}{ Number of points } & \multicolumn{2}{c}{ Original scheme } & \multicolumn{2}{c}{ Truncated scheme } \\
\cline { 2 - 5 } & BL-error & Order & BL-error & Order \\
\hline 40 & 0.19243 & NA & 0.074275 & NA \\
80 & 0.079672 & 1.2722 & 0.024212 & 1.6172 \\
160 & 0.028642 & 1.4759 & 0.0068855 & 1.8141 \\
320 & 0.0094434 & 1.6008 & 0.0018342 & 1.9084 \\
640 & 0.0029433 & 1.6818 & 0.00047321 & 1.9546 \\
1280 & 0.00088279 & 1.7373 & 0.00012017 & 1.9775 \\
\hline
\end{tabular}

and

$$
I_{1}(x)=\frac{1}{\pi} \int_{0}^{\pi} e^{x \cos (\theta)} \mathrm{d} \theta .
$$

Numerical simulations for this example are presented in Figure 2 with $\Delta x=1 / 40$ and the BL error and order of conference are presented in Table 2. For the order of convergence, the simulations are performed over the finite domain $x \in\left[10^{-2}, 20\right]$.

Example 6.7. In this example we consider fragmentation. We let $b(y, \cdot)=\frac{2}{y} \mathrm{~d} x$ and $a(x)=x$. As given in [54], this problem has an exact solution of

$$
\mu_{t}=(1+t)^{2} \exp (-x(1+t)) \mathrm{d} x
$$

Numerical simulations for this example are presented in Figure 3 with $\Delta x=1 / 40$ and the BL error and order of conference are presented in Table 2. Although convergence for the mass conserving fragmentation scheme is only shown for positive minimum mass, it still seems to perform well for the simulations below. Solving the fragmentation terms exactly leads to an $O\left(h^{2}\right)$ term in the last subinterval (where $y=x_{j}:=j \Delta x$ ). Explicitly, we have

$$
\alpha_{j}\left(x_{j}\right)=\frac{h}{x_{j}}+\frac{h^{2}}{x_{j}^{2}}
$$



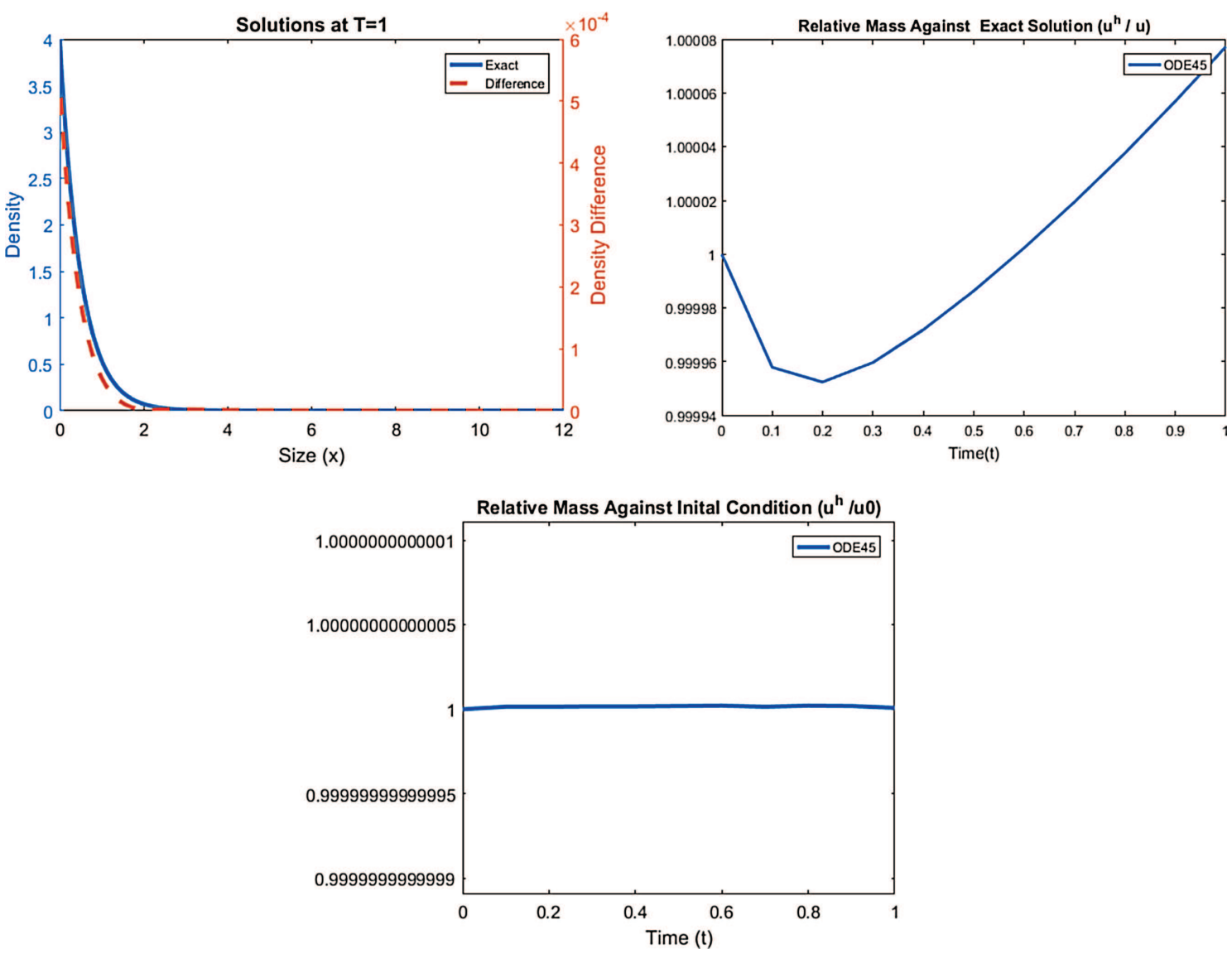

Figure 3. For Example 6.7 we present on the top left side the exact solution (solid line) and the absolute value of the difference between the exact and numerical solution (dashed-line). On the top right side we present the relative mass against the exact solution. On the bottom, we present the relative mass against the initial condition.

However, we noticed that for this last interval truncating the second term $\frac{h^{2}}{x_{j}^{2}}$, which is of order $O\left(h^{2}\right)$, we improve the scheme's performance. We present both the performance of the original scheme and the truncated scheme in Table 2. Simulations for Table 2 are performed over the finite domain $x \in[0,20]$.

Example 6.8. In this example, take $b(y, \cdot)=\frac{2}{y} \mathrm{~d} x$ and $a(x)=x^{2}$. Again, as given in [54], this problem has an exact solution of

$$
\mu_{t}=(1+2 t+2 t x) \exp (-x(1+x t)) \mathrm{d} x .
$$

Numerical simulations are presented for this example in Figure 4 with $\Delta x=1 / 40$. The BL error and order of convergence are presented in Table 3 . Simulations for Table 3 are performed over the finite domain $x \in[0,20]$.

Example 6.9. For this example, we demonstrate the performance of the scheme for a domain where the minimum size is positive. To this end, we truncate Example 6.7 above to the domain $\left[10^{-3}, 20\right]$ and use the kernel given by (6.4). Since the exact solution is not known for this equation, we compare to the solution given in Example 6.7. Though we do not compute any numerical orders of convergence, we point out the numerical and exact solutions in Figure 5 are very close. This simulation is again done with $\Delta x=\frac{1}{40}$. 

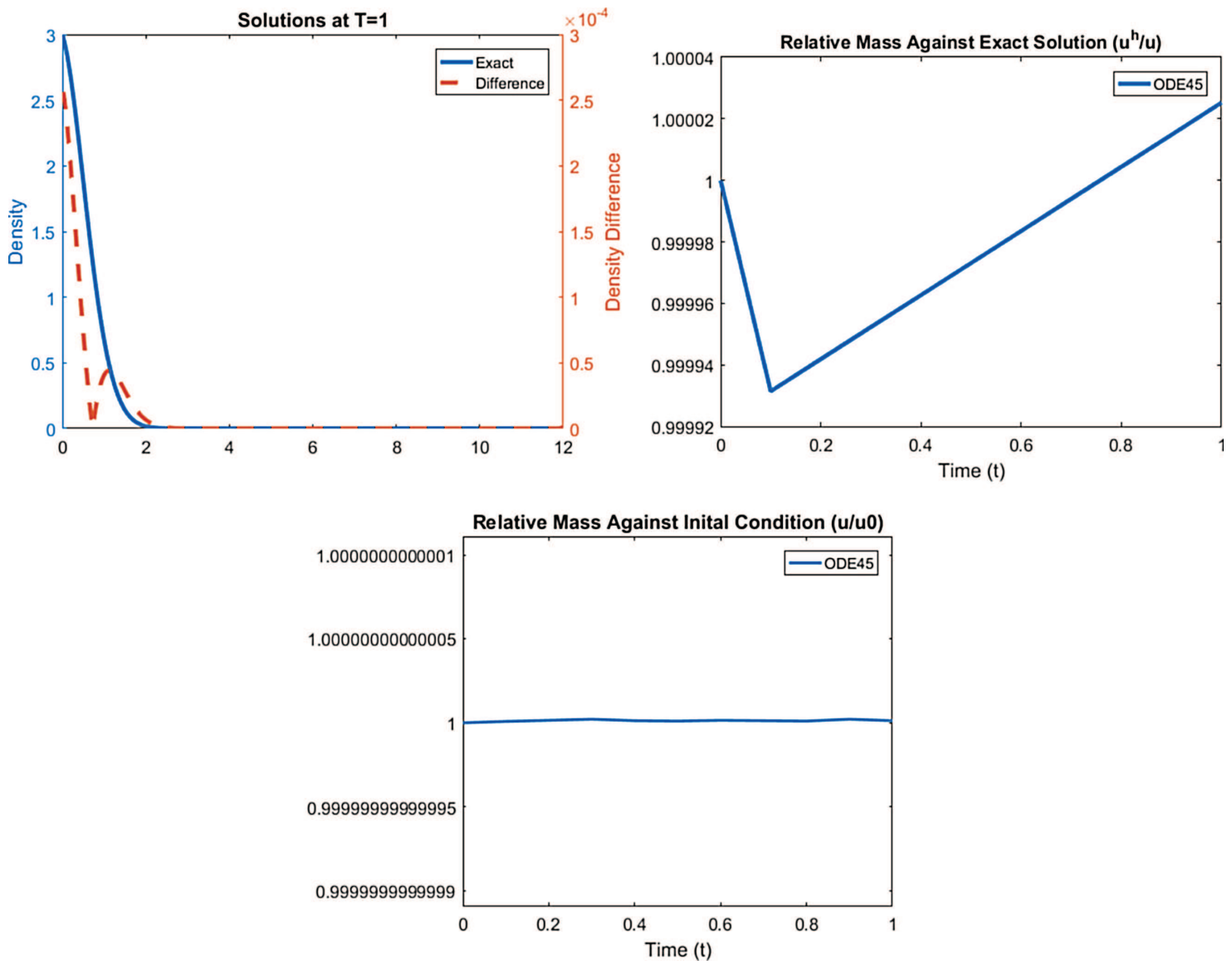

Figure 4. For Example 6.8 we present on the top left side the exact solution (solid line) and the absolute value of the difference between the exact and numerical solution (dashed-line). On the top right side we present the relative mass against the exact solution. On the bottom, we present the relative mass against the initial condition.

TABLE 3. Error and numerical order of convergence Example 6.8.

\begin{tabular}{lllll}
\hline \hline \multirow{2}{*}{ Number of points } & \multicolumn{2}{c}{ Original scheme } & \multicolumn{2}{c}{ Truncated scheme } \\
\cline { 2 - 5 } & BL-error & Order & \multicolumn{1}{c}{ BL-error } & Order \\
\hline 40 & 0.1471 & NA & 0.056501 & NA \\
80 & 0.041762 & 1.8165 & 0.014505 & 1.9617 \\
160 & 0.011112 & 1.9101 & 0.0036472 & 1.9917 \\
320 & 0.0028655 & 1.9553 & 0.00091301 & 1.9981 \\
640 & 0.00072752 & 1.9777 & 0.00022829 & 1.9998 \\
1280 & 0.00018324 & 1.9893 & 0.000057021 & 2.0013 \\
\hline
\end{tabular}



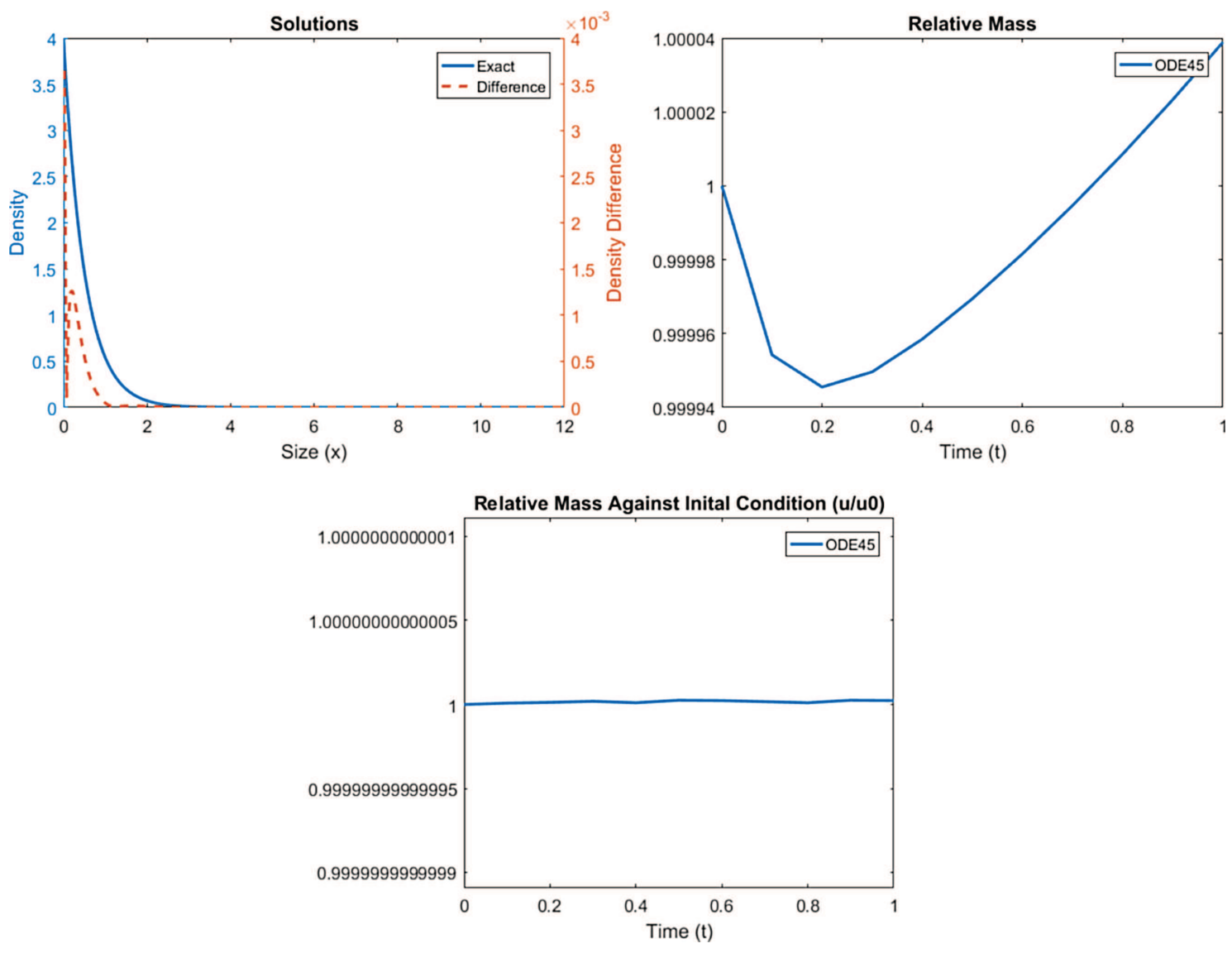

Figure 5. For Example 6.9 we present on the top left side the exact solution of Example 6.7 (solid line) and the absolute value of the difference between the exact and numerical solution (dashed-line). On the top right side we present the relative mass against the exact solution. On the bottom, we present the relative mass against the initial condition.

Example 6.10. In this example, we demonstrate what a discrete system would look like in our current frame work as well as provide an example of the results show in Theorem 5.1. We also demonstrate the mass conservation property of the coagulation terms of the scheme. The simulation is performed over the interval $[0,20]$ however, for clarity we zoom into the interval $[0,4]$. Take $\kappa(x, y) \equiv 1$ and $\mu_{0}=\delta_{0.2}+\delta_{0.4}$ (Fig. 6).

\section{Concluding Remarks}

In summary, we have presented a size-structured coagulation-fragmentation model formulated on the space of Radon measures endowed with the BL-norm. This model unifies the study of both the discrete and density based coagulation-fragmentation equations, both of which have been used in studying the dynamics of oceanic phytoplankton populations. We have shown, under biologically relevant assumptions (see e.g. [4] and the references therein), the model is well-posed using a fixed point approach discussed in recent papers [5,6]. We also established a regularity result that shows, under certain conditions on the model parameters, the solution to the model is absolutely continuous to the left of the characteristic curve emanating from the point $(0,0)$. This allows us to prove that any stationary solution of the model is absolutely continuous. This extends the result in [35] for 

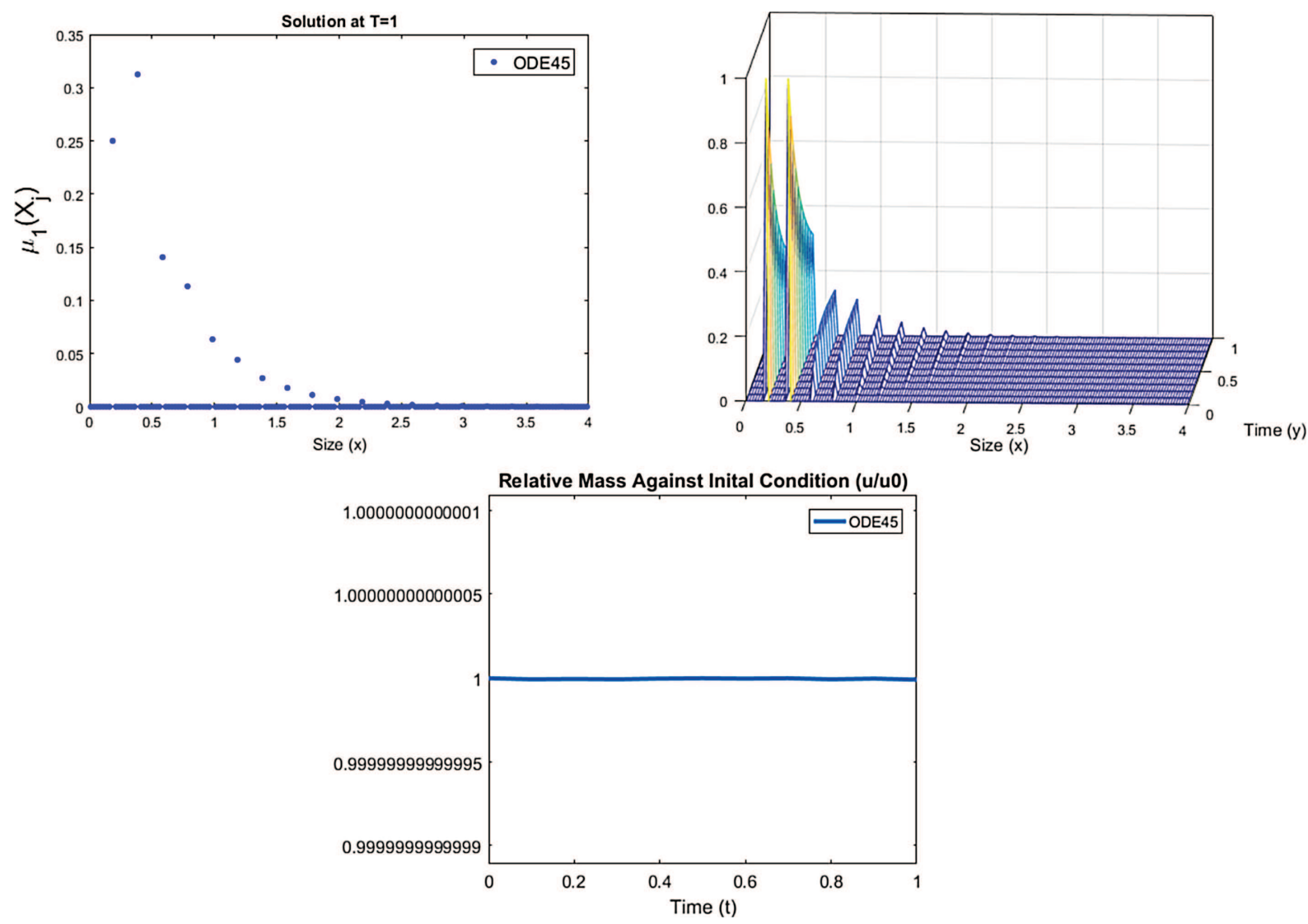

FiguRE 6. For Example 6.10 we present on the left side the numerical solution at time $T=1$. On the right side we present a mesh of the solution over time. On the bottom, we present the relative mass according to the initial condition over $[0,20]$.

structured population models without coagulation and fragmentation. Here, our proof differs from that in [35] since it relies on the implicit fixed point representation of the measure valued solution. Furthermore, we have shown how one obtains both the density and discrete coagulation-fragmentation equations from model (3.8). We also provided a semidiscrete method for approximating solutions to these equations and presented some numerical examples verifying our scheme. In these examples, we observed the semidiscrete scheme appears to have at best a second order convergence rate in the BL norm. In addition to the cases covered by our convergence proof, the scheme also seems to preform well in the case of a gelation coagulation kernel.

While the semidiscrete scheme presented in this paper is convergent and conserves mass, it does not take into account a growth term. In the future, we plan to develop and study fully discrete higher order schemes for the full model (3.8) that preserves solution non-negativity and mass (e.g. [14,46] in the space of integrable functions setting).

Acknowledgements. The research of A.S. Ackleh and R. Lyons is supported in part by funds from the R.P. Authement Eminent Scholar and Endowed Chair in Computational Mathematics at the University of Louisiana at Lafayette. 


\section{REFERENCES}

[1] A.S. Ackleh, Parameter estimation in a structured algal coagulation-fragmentation model. Nonlinear Anal.: Theory Methods App. 28 (1997) 837-854.

[2] A.S. Ackleh and K. Deng, A nonautonomous juvenile-adult model: well-posedness and long-time behavior via a comparison principle. SIAM J. Appl. Math. 69 (2009) 1644-1661.

[3] A.S. Ackleh and B.G. Fitzpatrick, Modeling aggregation and growth processes in an algal population model: analysis and computations. J. Math. Biol. 35 (1997) 480-502.

[4] A.S. Ackleh and R.L. Miller, A model for the interaction of phytoplankton aggregates and the environment: approximation and parameter estimation. Inverse Prob. Sci. Eng. 26 (2017) 152-182.

[5] A.S. Ackleh and N. Saintier, Well-posedness for a system of transport and diffusion equations in measure spaces. J. Math. Anal. App. 492 (2020) 1-32.

[6] A.S. Ackleh and N. Saintier, Diffusive limit to a selection-mutation equation with small mutation formulated on the space of measures. Discrete Continuous Dyn. Syst. Ser. B 26 (2021) 1469.

[7] A.S. Ackleh, K. Deng and X. Wang, Existence-uniqueness and monotone approximation for a phytoplankton-zooplankton aggregation model. Z. Angew. Math. Phys. 57 (2006) 733-749.

[8] A.S. Ackleh, J. Carter, K. Deng, Q. Huang, N. Pal and X. Yang, Fitting a structured juvenile-adult model for green tree frogs to population estimates from capture-mark-recapture field data. Bull. Math. Biol. 74 (2012) 641-665.

[9] A.S. Ackleh, R. Lyons and N. Saintier, Finite difference schemes for a structured population model in the space of measures. Math. Biosci. Eng. MBE 17 (2020) 747-775.

[10] J.M. Ball and J. Carr, The discrete coagulation-fragmentation equations: existence, uniqueness, and density conservation. J. Stat. Phys. 61 (1990) 203-234.

[11] J. Banasiak and W. Lamb, Coagulation, fragmentation and growth processes in a size structured population. Discrete Continuous Dyn. Syst.-Ser. B 11 (2009) 563-585.

[12] R.J.H. Beverton and S.J. Holt, On the dynamics of exploited fish populations. In: Fisheries and Food. Vol. XIX of Fishery Investigations Series II. Ministry of Agriculture (1957) 1-957.

[13] P.J. Blatz and A.V. Tobolsky, Note on the kinetics of systems manifesting simultaneous polymerization-depolymerization phenomena. J. Phys. Chem. 49 (1945) 77-80.

[14] J.P. Bourgade and F. Filbet, Convergence of a finite volume scheme for coagulation-fragmentation equations. Math. Comput. 77 (2008) 851-882.

[15] A.B. Burd and G.A. Jackson, Particle aggregation. Ann. Rev. Mar. Sci. 1 (2009) 65-90.

[16] J.A. Cañizo, Convergence to equilibrium for the discrete coagulation-fragmentation equations with detailed balance. J. Stat. Phys. 129 (2007) 1-26.

[17] J.A. Cañizo, J.A. Carrillo and S. Cuadrado, Measure solutions for some models in population dynamics. Acta Appl. Math. 123 (2013) 141-156.

[18] J. Carrillo, R.M. Colombo, P. Gwiazda and A. Ulikowska, Structured populations, cell growth and measure valued balance laws. J. Differ. Equ. 252 (2012) 3245-3277.

[19] J.M.C. Clark and V. Katsouros, Stably coalescent stochastic froths. Adv. Appl. Probab. 31 (1999) $199-219$.

[20] T. Debiec, M. Doumic, P. Gwiazda and E. Wiedemann, Relative entropy method for measure solutions of the growthfragmentation equation. SIAM J. Math. Anal. 50 (2018) 5811-5824.

[21] K. Deng and Y. Wu, Extinction and uniform strong persistence of a size-structured population model. Discrete Continuous Dyn. Syst. Ser. B 22 (2017) 831-840.

[22] R.M. Dudley, Distances of probability measures and random variables. In: Selected Works of RM Dudley. Springer (2010) $28-37$.

[23] A. Eibeck and W. Wagner, Approximative solution of the continuous coagulation-fragmentation equation. Stochastic Anal. App. 18 (2000) 921-948.

[24] J.H. Evers, S.C. Hille and A. Muntean, Mild solutions to a measure-valued mass evolution problem with flux boundary conditions. J. Differ. Equ. 259 (2015) 1068-1097.

[25] H. Federer, Geometric Measure Theory. Springer (2014).

[26] H. Federer, Colloquium lectures on geometric measure theory. Bull. Am. Math. Soc. 84 (1978) 291-338.

[27] R. Fortet and E. Mourier, Convergence de la Répartition Empirique Vers la Répartition Théorique. Annales scientifiques de l'École Normale Supérieure 70 (1953) 267-285.

[28] A.K. Giri, J. Kumar and G. Warnecke, The continuous coagulation equation with multiple fragmentation. J. Math. Anal. App. 374 (2011) 71-87.

[29] A.K. Giri, P. Laurençot and G. Warnecke, Weak solutions to the continuous coagulation equation with multiple fragmentation. Nonlinear Anal. Theory Methods App. 75 (2012) 2199-2208.

[30] P. Gwiazda and A. Marciniak-Czochra, Structured population equations in metric spaces. J. Hyperbolic Differ. Equ. 07 (2010) 733-773.

[31] P. Gwiazda, T. Lorenz and A. Marciniak-Czochra, A Nonlinear structured population model: Lipschitz continuity of measurevalued solutions with respect to model ingredients. J. Differ. Equ. 248 (2010) 2703-2735.

[32] P. Gwiazda, A. Marciniak-Czochra and H.R. Thieme, Measures under the flat norm as ordered normed vector space. Positivity $22(2017)$ 105-138. 
[33] O.J. Heilmann, Analytical solutions of Smoluchowski's coagulation equation. J. Phys. A25 (1992) $3763-3771$.

[34] J. Jabłoński and A. Marciniak-Czochra, Efficient algorithms computing distances between Radon measures on $\mathbb{R}$. Preprint t arXiv:1304.3501 (2013).

[35] J. Jabłoński and D. Wrzosek, Measure-valued solutions to size-structured population model of prey controlled by optimally foraging predator harvester. Math. Models Methods Appl. Sci. 29 (2019) 1657-1689.

[36] G.A. Jackson, A model of the formation of marine algal flocs by physical coagulation processes. Deep Sea Res. Part A. Oceanogr. Res. Papers 37 (1990) 1197-1211.

[37] G.A. Jackson and S.E. Lochmann, Effect of coagulation on nutrient and light limitation of an algal bloom. Limnol. Oceanogr. 37 (1992) 77-89.

[38] I. Jeon, Existence of getting solutions for coagulation-fragmentation equations. Commun. Math. Phys. 194 (1998) 541-567.

[39] D.D. Keck and D.M. Bortz, Numerical simulation of solutions and moments of the Smoluchowski coagulation equation. Preprint arXiv:1312.7240 (2013).

[40] W. Lamb, Existence and uniqueness results for the continuous coagulation and fragmentation equation. Math. Models Methods Appl. Sci. 27 (2004) 703-721.

[41] A. Lasota, J. Myjak and T. Szarek, Markov operators with a unique invariant measure. J. Math. Anal. App. 276 (2002) 343-356.

[42] P. Laurençot, On a class of continuous coagulation-fragmentation equations. J. Differ. Equ. 167 (2000) 245-274.

[43] P. Laurençot and S. Mischler, Global existence for the discrete diffusive coagulation-fragmentation equations in $L^{1}$. Rev. Mat. Iberoam. 18 (2002) 731-745.

[44] P. Laurençot and S. Mischler, From the discrete to the continuous coagulation fragmentation equations. Proc. R. Soc. Edinburgh Sect. A: Math. 132 (2002) 1219-1248.

[45] P. Laurençot and S. Mischler, On coalescence equations and related models, edited by P. Degond, L. Pareschi, G. Russo. In: Modeling and Computational Methods for Kinetic Equations. Birkhäuser, Boston (2004) 321-356.

[46] H. Liu, R. Gröpler and G. Warnecke, A high order positivity preserving DG method for coagulation-fragmentation equations. SIAM J. Sci. Comput. 41 (2019) 448-465.

[47] D.J. McLaughlin, W. Lamb and A.C. McBride, An existence and uniqueness result for a coagulation and multiple-fragmentation equation. SIAM J. Math. Anal. 28 (1997) 1173-1190.

[48] Z.A. Melzak, A scalar transport equation. Trans. Am. Math. Soc. 85 (1957) 547-560.

[49] H. Müller, Zur Allgemeinen Theorie der Raschen Koagulation. Fortschrittsberichte ber Kolloide und Polymere 27 (1928) $223-250$.

[50] J.R. Norris, Smoluchowski's coagulation equation: uniqueness, non-uniqueness and a hydrodynamic limit for the stochastic coalescent. Ann. Appl. Probab. 9 (1999) 78-109.

[51] D. Pauly and G.R. Morgan, Length-Based Methods in Fisheries Research. WorldFish 13 (1987).

[52] W.E. Ricker, Stock and recruitment. J. Fisheries Board Can. 11 (1954) 559-623.

[53] R. Rudnicki and R. Wieczorek, Fragmentation-coagulation models of phytoplankton. Bull. Polish Acad. Sci. Math. 54 (2006) $175-191$.

[54] R. Singh, J. Saha and J. Kumar, Adomian decomposition method for solving fragmentation and aggregation population balance equations. J. Appl. Math. Comput. 48 (2014) 265-292.

[55] M. Smoluchowski, Drei Vorträge über Diffusion, Brownsche Molekularbewegung und Koagulation von Kolloidteilchen. Phys. Z. 17 (1916) 557-571, 585-599.

[56] I.W. Stewart, A global existence theorem for the general coagulation-fragmentation equation with unbounded kernels. Math. Methods Appli. Sci. 11 (1989) 627-648.

\section{Subscribe to Open (S2O)}

\section{A fair and sustainable open access model}

This journal is currently published in open access under a Subscribe-to-Open model (S2O). S2O is a transformative model that aims to move subscription journals to open access. Open access is the free, immediate, online availability of research articles combined with the rights to use these articles fully in the digital environment. We are thankful to our subscribers and sponsors for making it possible to publish this journal in open access, free of charge for authors.

\section{Please help to maintain this journal in open access!}

Check that your library subscribes to the journal, or make a personal donation to the $\mathrm{S} 2 \mathrm{O}$ programme, by contacting subscribers@edpsciences.org

More information, including a list of sponsors and a financial transparency report, available at: https://www. edpsciences.org/en/maths-s2o-programme 